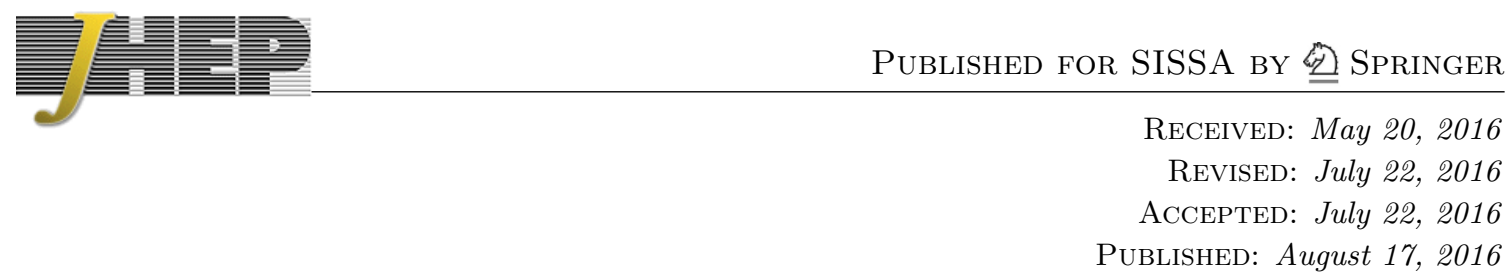

\title{
Geometry of the scalar sector
}

\author{
Rodrigo Alonso, ${ }^{a}$ Elizabeth E. Jenkins ${ }^{a, b}$ and Aneesh V. Manohar ${ }^{a, b}$ \\ ${ }^{a}$ Department of Physics, University of California at San Diego, \\ La Jolla, CA 92093, U.S.A. \\ ${ }^{b}$ CERN TH Division, \\ CH-1211 Geneva 23, Switzerland \\ E-mail: ralonsod@ucsd.edu, ejenkins@ucsd.edu, amanohar@ucsd.edu
}

ABSTRACT: The $S$-matrix of a quantum field theory is unchanged by field redefinitions, and so it only depends on geometric quantities such as the curvature of field space. Whether the Higgs multiplet transforms linearly or non-linearly under electroweak symmetry is a subtle question since one can make a coordinate change to convert a field that transforms linearly into one that transforms non-linearly. Renormalizability of the Standard Model (SM) does not depend on the choice of scalar fields or whether the scalar fields transform linearly or non-linearly under the gauge group, but only on the geometric requirement that the scalar field manifold $\mathcal{M}$ is flat.

Standard Model Effective Field Theory (SMEFT) and Higgs Effective Field Theory (HEFT) have curved $\mathcal{M}$, since they parametrize deviations from the flat SM case. We show that the HEFT Lagrangian can be written in SMEFT form if and only if $\mathcal{M}$ has a SU(2) ${ }_{L} \times$ $\mathrm{U}(1)_{Y}$ invariant fixed point. Experimental observables in HEFT depend on local geometric invariants of $\mathcal{M}$ such as sectional curvatures, which are of order $1 / \Lambda^{2}$, where $\Lambda$ is the EFT scale. We give explicit expressions for these quantities in terms of the structure constants for a general $\mathcal{G} \rightarrow \mathcal{H}$ symmetry breaking pattern. The one-loop radiative correction in HEFT is determined using a covariant expansion which preserves manifest invariance of $\mathcal{M}$ under coordinate redefinitions. The formula for the radiative correction is simple when written in terms of the curvature of $\mathcal{M}$ and the gauge curvature field strengths. We also extend the CCWZ formalism to non-compact groups, and generalize the HEFT curvature computation to the case of multiple singlet scalar fields.

Keywords: Effective field theories, Gauge Symmetry, Sigma Models

ARXIV EPRINT: 1605.03602 


\section{Contents}

1 Introduction 1

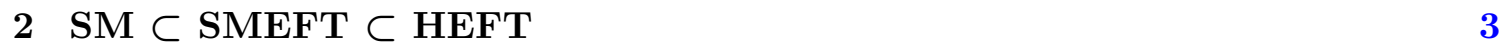

$2.1 O(4)$ fixed point 8

2.2 SMEFT 9

$\begin{array}{lll}2.3 \text { HEFT } & 10\end{array}$

3 Covariant formalism for curved scalar field space 13

3.1 Scalar fields on a curved manifold $\mathcal{M} \quad 13$

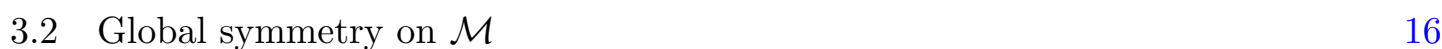

$\begin{array}{lll}3.3 & \text { Local symmetry on } \mathcal{M} & 17\end{array}$

4 CCWZ and non-compact groups $\quad 20$

$\begin{array}{lll}4.1 & \text { Matter fields } & 27\end{array}$

$\begin{array}{ll}4.2 & \text { Sectional curvature }\end{array}$

5 The Standard Model and custodial symmetry violation 29

$6 \quad$ HEFT with multiple singlet scalar bosons 32

7 Conclusions $\quad 34$

A Exponential parametrization of the $O(N)$ model 35

B One-loop renormalization of HEFT 35

C Non-reductive cosets

C.1 Example of a non-reductive coset 40

\section{Introduction}

Current experimental data is consistent with the predictions of the Standard Model (SM) with a light Higgs boson of mass $\sim 125 \mathrm{GeV}$. The measured properties of the Higgs boson agree with SM predictions, but the current experimental accuracy of measured singleHiggs boson couplings is only at the level of $\sim 10 \%$, and no multi-Higgs boson couplings have been measured directly. It is important to consider generalizations of the SM with additional parameters in order to quantify the accuracy to which the SM is valid or to detect deviations from SM predictions.

Over the past 40 years, many theoretical ideas have been proposed for the underlying mechanism of electroweak symmetry breaking. Theories that survive must be consistent with the currently observed pattern of electroweak symmetry breaking, which is 
well-described by the SM. A general model-independent analysis of electroweak symmetry breaking can be performed using effective field theory (EFT) techniques. Assuming there are no additional light particles beyond those of the SM at the electroweak scale $v \sim 246 \mathrm{GeV}$, the EFT has the same field content as the SM. There are two main EFTs used in the literature, the Standard Model Effective Field Theory (SMEFT) and Higgs Effective Field Theory (HEFT). In this paper, we make the relationship between these two theories precise.

The Higgs boson $h$ of the SM is a neutral $0^{+}$scalar particle. In the SM Lagrangian, it appears in a complex scalar field $H$, which transforms as $\mathbf{2}_{1 / 2}$ under the $\mathrm{SU}(2)_{L} \times \mathrm{U}(1)_{Y}$ electroweak gauge symmetry. An oft-stated goal of the precision Higgs physics program is to test whether (a) the Higgs boson transforms as part of a complex scalar doublet which mixes linearly under $\mathrm{SU}(2)_{L} \times \mathrm{U}(1)_{Y}$ with the three "eaten" Goldstone bosons $\boldsymbol{\varphi}$, or (b) whether the Higgs field is a singlet radial direction which does not transform under the electroweak symmetry. In case (b), the three Goldstone modes $\varphi$ transform non-linearly amongst themselves under the electroweak symmetry, in direct analogy to pions in QCD chiral perturbation theory, and do not mix with the singlet Higgs field. In case (a), there are relations between Higgs boson and Goldstone boson (i.e. longitudinal gauge boson) interactions, whereas in case (b), no relations are expected in general. An objective of this paper is to explore the distinction between these two pictures for Higgs boson physics.

The properties of the scalar sector of the SM and its EFT generalizations can be clarified by studying it from a geometrical point of view [1]. The scalar fields define coordinates on a scalar manifold $\mathcal{M}$. The geometry of $\mathcal{M}$ is invariant under coordinate transformations, which are scalar field redefinitions. The quantum field theory $S$-matrix also is invariant under scalar field redefinitions, so it depends only on coordinate-independent properties of $\mathcal{M}$. Consequently, experimentally measured quantities depend only on the geometric invariants of $\mathcal{M}$, such as the curvature. Formulating physical observables geometrically avoids arguments based on a particular choice of fields. It also allows us to correctly pose and answer the question of whether the Higgs boson transforms linearly or non-linearly under the electroweak gauge symmetry. Further, a geometric analysis gives a better understanding of the structure of the theory and its coordinate-invariant properties.

The UV theory can have additional states, such as massive meson excitations in the case of theories with strong dynamics. At low energies, the EFT interactions in the electroweak symmetry breaking sector are described by a Lagrangian with scalar degrees of freedom on some manifold $\mathcal{M}$, with the Lagrangian expanded in gradients of the scalar fields. The geometric description captures the features of the UV dynamics needed to make predictions for experiments at energies below the scale of new physics.

The geometrical structure of non-linear sigma models has been worked out over many years, mainly in the context of supersymmetric sigma models (see e.g. [2-12]). The applications to the SM Higgs sector presented here are new, and they provide a better understanding of the structure of HEFT and the search for signals of new physics through the couplings of the Higgs boson.

Some of the results in this paper have already been given in ref. [1]. Here we provide more explanation of the results presented there as well as details of explicit calculations in 
that work, including the derivation of the one-loop effective action for a curved scalar manifold $\mathcal{M}$. For most of the paper, we will assume that the scalar sector has an enlarged global symmetry, known as custodial symmetry. Also note that we will usually treat the scalar sector in the ungauged case, referring to the scalar fields as Higgs and Goldstone bosons. The gauged version of the theory follows immediately by replacing ordinary derivatives by gauge covariant derivatives. In the gauged case, the Goldstone bosons are eaten via the Higgs mechanism, becoming the longitudinal polarization states of the massive electroweak gauge bosons. Thus, the Higgs-Goldstone boson relations we refer to are in fact relations between the couplings of the Higgs boson and the three longitudinal gauge boson states $W_{L}^{ \pm}$and $Z_{L}[13-15]$.

The organization of the paper is as follows. The relationship between the SM, SMEFT and HEFT is discussed in section 2 from a geometrical point of view. It is shown that SMEFT is a special case of HEFT when $\mathcal{M}$ is expanded about an $O(4)$ invariant fixed point. Further, it is shown that the existence of such an $O(4)$ invariant fixed point is a necessary and sufficient condition for the existence of a choice of scalar fields such that the Higgs field transforms linearly under the electroweak gauge symmetry. Section 3 presents the covariant formalism for curved scalar field space. We discuss global and gauge symmetries in terms of Killing vectors of the scalar manifold, and we derive the one-loop correction to the effective action for curved $\mathcal{M}$. In section 4 , the geometric formulation of $\mathcal{G} / \mathcal{H}$ theories is connected with the standard coordinates of CCWZ. We give formulæ for the curvature tensor in terms of field strengths for a general sigma model. We also discuss the extension of the CCWZ standard coordinates to non-compact groups. As shown in ref. [1], the sign of deviations from SM values of Higgs boson-longitudinal gauge boson scattering amplitudes is controlled by sectional curvatures in HEFT. For $\mathcal{G} / \mathcal{H}$ theories based on compact groups, these sectional curvatures are typically positive. We compute the sectional curvature, and show that in certain cases, it can be negative. In section 5 , we briefly discuss the SM and custodial symmetry violation, and the relation between the SM scalar manifold and the configuration space of a rigid rotator. Section 6 generalizes HEFT to the case of multiple singlet Higgs bosons. Finally, section 7 provides our conclusions. Additional formulae are provided in the appendices, including intermediate steps in the computation of the one-loop correction to HEFT given in refs. [1, 16], and discussion of the complications for non-reductive cosets.

\section{$2 \mathrm{SM} \subset \mathrm{SMEFT} \subset \mathrm{HEFT}$}

In this section, we discuss the scalar sector of the SM and its EFT generalizations, SMEFT and HEFT, as well as the relationship between these three theories. We begin with a summary of the scalar sector of the SM.

The SM scalar Lagrangian (with the gauge fields turned off) is

$$
L=\partial_{\mu} H^{\dagger} \partial_{\mu} H-\lambda\left(H^{\dagger} H-\frac{v^{2}}{2}\right)^{2} .
$$

This scalar Lagrangian is the most general $\mathrm{SU}(2)_{L} \times \mathrm{U}(1)_{Y}$ invariant Lagrangian with terms of dimension $\leq 4$ built out of a Higgs doublet $H$ that transforms as $\mathbf{2}_{1 / 2}$ under 
$\mathrm{SU}(2)_{L} \times \mathrm{U}(1)_{Y}$. As is well-known, the SM scalar sector has an enhanced global custodial symmetry group $O(4) \sim \mathrm{SU}(2)_{L} \times \mathrm{SU}(2)_{R}$. This global symmetry can be made manifest by writing the SM complex scalar doublet field $H$ in terms of four real scalar fields,

$$
H \equiv \frac{1}{\sqrt{2}}\left[\begin{array}{c}
\phi^{2}+i \phi^{1} \\
\phi^{4}-i \phi^{3}
\end{array}\right] .
$$

Substitution in eq. (2.1) yields the Lagrangian

$$
L=\frac{1}{2} \partial_{\mu} \boldsymbol{\phi} \cdot \partial_{\mu} \phi-\frac{\lambda}{4}\left(\boldsymbol{\phi} \cdot \boldsymbol{\phi}-v^{2}\right)^{2},
$$

where $\phi=\left(\phi^{1}, \phi^{2}, \phi^{3}, \phi^{4}\right)$. Lagrangian eq. (2.3) is invariant under $\mathcal{G}=O(4)$ global symmetry transformations

$$
\phi \rightarrow O \phi, \quad O^{T} O=1 .
$$

The scalar field $\phi$ transforms linearly as the four-dimensional vector representation of the global symmetry group $\mathcal{G}=O(4)$. The minimum of the potential is the three-sphere $S^{3}$ of radius $v$,

$$
\langle\phi \cdot \phi\rangle=v^{2}
$$

which is the Goldstone boson vacuum submanifold of the SM. The radius of the sphere, $v \sim 246 \mathrm{GeV}$, is fixed by the gauge boson masses. It is conventional to choose the vacuum expectation value

$$
\langle\phi\rangle=v\left[\begin{array}{l}
0 \\
0 \\
0 \\
1
\end{array}\right]
$$

and expand the Lagrangian about this vacuum state in the shifted fields $\phi^{4} \equiv v+\mathrm{h}$ and $\phi^{a} \equiv \varphi^{a}, a=1,2,3$,

$$
\phi=\left[\begin{array}{c}
\varphi^{1} \\
\varphi^{2} \\
\varphi^{3} \\
v+\mathrm{h}
\end{array}\right], \quad H=\frac{1}{\sqrt{2}}\left[\begin{array}{c}
\varphi^{2}+i \varphi^{1} \\
v+\mathrm{h}-i \varphi^{3}
\end{array}\right]
$$

The vacuum expectation value $\langle\phi\rangle$ spontaneously breaks the global symmetry group $\mathcal{G}=$ $O(4)$ to the unbroken global symmetry group $\mathcal{H}=O(3)$. The Goldstone bosons $\varphi^{a}, a=$ $1,2,3$, transform as a triplet under the unbroken global symmetry, whereas $\mathrm{h}$ transforms as a singlet. We will refer to both the enlarged global symmetries $\mathcal{G}=O(4) \sim \mathrm{SU}(2)_{L} \times \mathrm{SU}(2)_{R}$ and $\mathcal{H}=O(3) \sim \mathrm{SU}(2)_{V}$ as custodial symmetries. The unbroken global symmetry group $\mathcal{H}$ leads to the relation $M_{W}=M_{Z} \cos \theta_{W}$, which is a successful prediction of the SM. The experimental success of this gauge boson mass relation implies that custodial symmetry is a good approximate symmetry of the SM. 
The Lagrangian eq. (2.3) in terms of shifted fields eq. (2.7) becomes

$$
L=\frac{1}{2} \partial_{\mu} \boldsymbol{\varphi} \cdot \partial_{\mu} \boldsymbol{\varphi}+\frac{1}{2}\left(\partial_{\mu} \mathrm{h}\right)^{2}-\frac{\lambda}{4}\left(\mathrm{~h}^{2}+2 \mathrm{~h} v+\boldsymbol{\varphi} \cdot \boldsymbol{\varphi}\right)^{2} .
$$

The singlet $\mathrm{h}$ is the physical Higgs field with mass

$$
m_{\mathrm{h}}^{2}=2 \lambda v^{2}
$$

whereas the Goldstone bosons are strictly massless. In the gauged theory, the three Goldstone bosons $\varphi^{a}$ of the $\mathcal{G} \rightarrow \mathcal{H}$ global symmetry breakdown are "eaten" via the Higgs mechanism, becoming the longitudinal polarization states of the massive $W^{ \pm}$and $Z$ gauge bosons. Note that the $O(4)$-invariant potential $V(\mathrm{~h}, \varphi)$ depends on an $O(4)$-invariant combination of both $\mathrm{h}$ and $\varphi$.

Equating the scalar kinetic energy term in eq. (2.8) with

$$
L_{\mathrm{KE}}=\frac{1}{2} g_{i j}(\phi)\left(\partial_{\mu} \phi^{i}\right)\left(\partial^{\mu} \phi^{j}\right), \quad i, j=1,2,3,4,
$$

defines the scalar metric $g_{i j}^{\mathrm{SM}}(\phi)=\delta_{i j}$ for the SM scalar manifold $\mathcal{M}$ with coordinates given by the scalar fields $\phi^{i}$. Distances on $\mathcal{M}$ are determined by $d s^{2}=g_{i j}(\phi) d \phi^{i} d \phi^{j}$.

The four-dimensional SM scalar manifold $\mathcal{M}=\mathbb{R}^{4}$ is shown in figure 1 . The $O(4)$ symmetry acts by rotations. The minimum of the potential is the solid red curve, and forms the three-dimensional Goldstone boson submanifold $S^{3}$ of radius $v$. The parameterization eq. (2.7) is a Cartesian coordinate system for $\mathcal{M}$ centered on the vacuum (black dot), where $\mathrm{h}$ is the horizontal direction, and $\varphi^{a}, a=1,2,3$, are the three other directions orthogonal to h. The angular coordinates of $S^{3}$ are $\varphi^{a} / v$. The $O(4)$ symmetry acts linearly on $\left(\varphi^{1}, \varphi^{2}, \varphi^{3}, v+\mathrm{h}\right)$.

In Cartesian coordinates, it seems intuitively clear that $\varphi^{a}$ and $\mathrm{h}$ interactions are related, given that the four scalar fields belong to the same Higgs doublet eq. (2.2). However, the precise relation is subtle. In order to understand this point better, it is instructive to express the SM Lagrangian eq. (2.3) in polar coordinates as well.

In polar coordinates, ${ }^{1}$

$$
\boldsymbol{\phi}=(v+h) \boldsymbol{n}(\pi), \quad \boldsymbol{n} \cdot \boldsymbol{n}=1,
$$

where $(v+h)$ is the magnitude of $\boldsymbol{\phi}$, and $\boldsymbol{n}(\pi) \in S^{3}$ is a four-dimensional unit vector. The four shifted scalar fields consist of the three dimensionless angular coordinates $\bar{\pi}^{a}=\pi^{a} / v$ (the direction of $\boldsymbol{n}(\pi)$ on $S^{3}$ ), and the radial coordinate $h$. The SM Lagrangian in polar coordinates is

$$
L=\frac{1}{2}(v+h)^{2}\left(\partial_{\mu} \boldsymbol{n}\right)^{2}+\frac{1}{2}\left(\partial_{\mu} h\right)^{2}-\frac{\lambda}{4}\left(h^{2}+2 v h\right)^{2} .
$$

An advantage of expressing the SM Lagrangian in polar coordinates is that the three Goldstone boson fields of $\boldsymbol{n}(\pi)$ are derivatively coupled. In addition, the scalar potential in

\footnotetext{
${ }^{1}$ We use $\mathrm{h}, \varphi$ for the fields in Cartesian coordinates, and $h, \pi$ (or $\left.h, \mathbf{n}\right)$ in polar coordinates.
} 

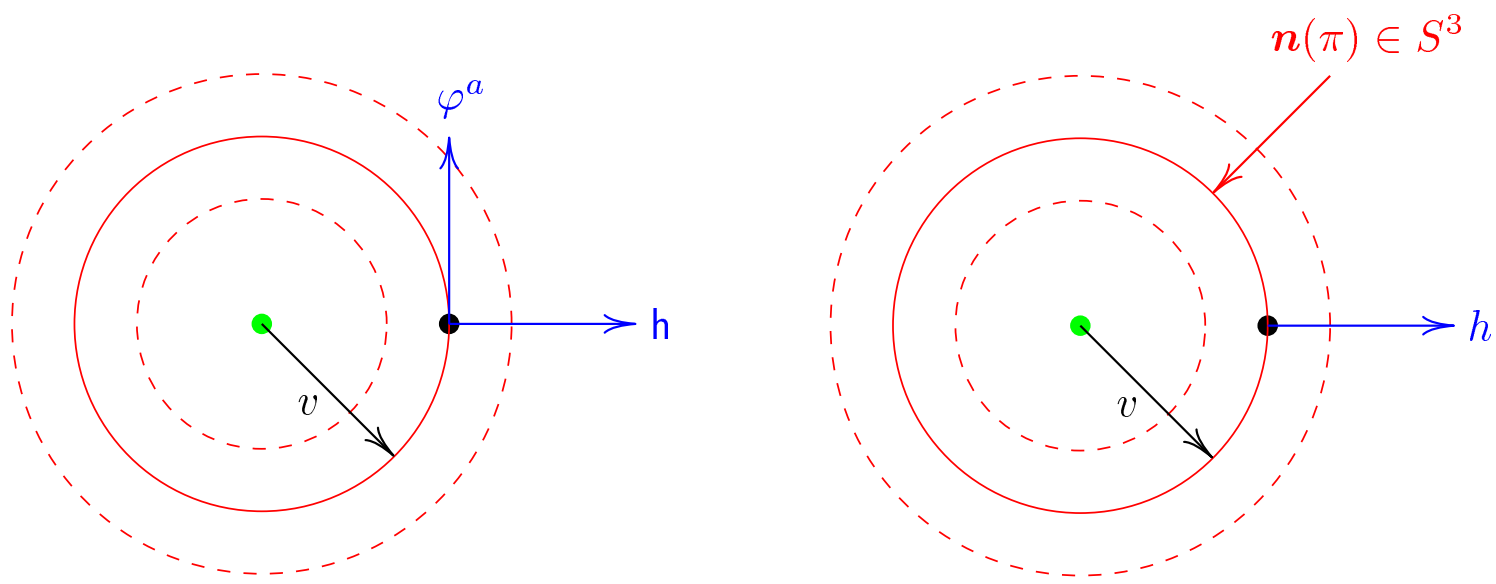

Figure 1. Two-dimensional depiction of the four-dimensional scalar manifold $\mathcal{M}=\mathbb{R}^{4}$ of the SM. The SM vacuum is the black dot shown in the figure. The origin (green dot) is an $O(4)$ invariant fixed point. The left and right diagrams show the fields in Cartesian and polar coordinates, respectively. $O(4)$ symmetry acts linearly on the Cartesian coordinates. In polar coordinates, $h$ is $O(4)$-invariant, and the angular coordinates $\boldsymbol{n}(\pi)$ transform non-linearly under the $O(4)$ symmetry. The scalar manifold $\mathcal{M}$ is flat, so the scale $\Lambda$ setting the curvature is formally infinite.

polar coordinates only depends on the radial coordinate $h$, whereas in Cartesian coordinates it depends on all four scalar fields.

The $O(4)$ symmetry transformations of $\mathcal{M}$ in polar coordinates are

$$
h \rightarrow h, \quad \boldsymbol{n} \rightarrow O \boldsymbol{n},
$$

so the Higgs field $h$ is invariant under $O(4)$ transformations, and $\boldsymbol{n}$ transforms linearly by an orthogonal transformation that preserves the constraint $\boldsymbol{n} \cdot \boldsymbol{n}=1$. Due to the constraint, however, only three of the four components of $\boldsymbol{n}$ are independent. Without loss of generality, one can take the first three components of $\boldsymbol{n}$ to be the independent components. Then, the fourth component $n^{4}$ is a non-linear function of the independent components $n^{1,2,3}$. The non-linear constraint $\boldsymbol{n} \cdot \boldsymbol{n}=1$ turns the linear $O(4)$ transformation on $\boldsymbol{n}$ into a non-linear transformation when written in terms of unconstrained fields. Thus, the $O(4)$ transformation on the three independent angular coordinates $\pi^{a} / v$ is a non-linear transformation.

Many different parameterizations of $\boldsymbol{n}(\pi)$ in terms of the independent unconstrained coordinates $\pi^{a} / v$ are possible. Two natural non-linear parameterizations are the square root parameterization and the exponential parameterization, which are defined by

$$
\boldsymbol{n}(\pi)=\frac{1}{v}\left[\begin{array}{c}
\pi^{1} \\
\pi^{2} \\
\pi^{3} \\
\sqrt{v^{2}-\boldsymbol{\pi} \cdot \boldsymbol{\pi}}
\end{array}\right]
$$


and

$$
\boldsymbol{n}(\pi)=\exp \left(\frac{1}{v}\left[\begin{array}{cccc}
0 & 0 & 0 & \pi^{1} \\
0 & 0 & 0 & \pi^{2} \\
0 & 0 & 0 & \pi^{3} \\
-\pi^{1} & -\pi^{2} & -\pi^{3} & 0
\end{array}\right]\right)\left[\begin{array}{l}
0 \\
0 \\
0 \\
1
\end{array}\right]
$$

respectively. For most of this paper, we use the exponential parameterization for $\boldsymbol{n}(\pi)$ since it corresponds to the standard coordinates of CCWZ.

Rotations in the 12, 13 and 23 planes act linearly on $\left(n^{1}, n^{2}, n^{3}\right)$, and leave $n^{4}$ invariant. However, rotations in the 14,24 and 34 planes mix $\left(n^{1}, n^{2}, n^{3}\right)$ and $n^{4}$. For example, a 14 rotation gives

$$
\delta n^{1}=\delta \theta n^{4}, \quad \delta n^{2}=0, \quad \delta n^{3}=0, \quad \delta n^{4}=-\delta \theta n^{1} .
$$

In terms of the independent unconstrained coordinates $\pi^{a}$ of the square root parameterization, 12, 13 and 23 rotations act linearly, but a 14 rotation gives

$$
\delta \pi^{1}=\delta \theta \sqrt{v^{2}-\boldsymbol{\pi} \cdot \boldsymbol{\pi}}, \quad \delta \pi^{2}=0, \quad \delta \pi^{3}=0 .
$$

The $O(4)$ transformation eq. (2.17) is non-linear. Consequently, eq. (2.13) is called a non-linear transformation, since it is non-linear when written in terms of unconstrained coordinates $\left(\pi^{1}, \pi^{2}, \pi^{3}\right)$.

In polar coordinates, $\boldsymbol{n}$ and $h$ are very different objects, and it is not at all obvious that $\boldsymbol{n}$ and $h$ interactions are related. Nevertheless, all we have done is switch from Cartesian coordinates $\left\{\varphi^{a}, \mathrm{~h}\right\}$ to polar coordinates $\left\{\pi^{a}, h\right\}$ while keeping the Lagrangian fixed. This change of coordinates does not affect physical observables such as $S$-matrix elements. Any relations that exist amongst physical observables must be present irrespective of the choice of coordinates.

We have summarized the standard analysis of the SM in Cartesian and polar coordinates. In Cartesian coordinates, the Higgs field $\mathrm{h}$ and the three Goldstone fields $\varphi^{a}$ form a four-dimensional representation which transforms linearly under $O(4)$. In polar coordinates, the Higgs field $h$ is an $O(4)$ singlet or invariant, and the three Goldstone bosons $\pi^{a}$ parameterizing the $S^{3}$ unit vector $\boldsymbol{n}(\pi)$ transform among themselves under the non-linear $O(4)$ transformation law eq. (2.13). The Higgs boson field $h$ in polar coordinates is not the same field as the Higgs boson field $h$ in Cartesian coordinates. The relation between the two Higgs boson fields is

$$
(v+h)^{2}=(v+\mathrm{h})^{2}+\varphi \cdot \varphi
$$

so that

$$
h=\mathrm{h}+\frac{\varphi \cdot \varphi}{2 v}-\frac{1}{2} \frac{\mathrm{h} \varphi \cdot \varphi}{v^{2}}+\ldots
$$

By the Lehmann-Symanzik-Zimmermann (LSZ) reduction formula, $h$ and $\mathrm{h}$ give the same $S$-matrix, and both are perfectly acceptable choices for the Higgs boson field. ${ }^{2}$

\footnotetext{
${ }^{2}$ The nomenclature "the Higgs field" is misleading, since there is no unique choice for the Higgs field.
} 


\section{$2.1 O(4)$ fixed point}

We now return to the question of whether the Higgs field transforms linearly or non-linearly under the electroweak gauge symmetry, and whether interactions of the Higgs boson and the three Goldstone bosons (i.e. longitudinal gauge boson polarizations) are related. As we have just seen, this question is not well-posed in the SM, since the answer depends on the choice of coordinates. However, it is intuitively clear that there is an underlying relationship between the couplings of the Higgs and Goldstone bosons in the SM that does not remain valid in the general context of HEFT. We need to formulate any coupling relations in a coordinate-invariant way. There are two conditions which make the SM special - (i) there is a point $\phi=\mathbf{0}$ (or $H=\mathbf{0}$ ) of $\mathcal{M}$ which is an $O(4)$ invariant fixed point, and (ii) the scalar manifold $\mathcal{M}$ is flat, i.e. it has a vanishing Riemann curvature tensor. ${ }^{3}$ As we now see, relations in the SM between the couplings of the Higgs boson and the three Goldstone bosons arise from these two conditions which are no longer true in HEFT in general.

We first analyze whether the Higgs field is part of a multiplet that transforms linearly under the $O(4)$ symmetry. Even in the SM, the answer to this question depends on the choice of coordinates. The coordinate-invariant formulation of the question is: does there exist a choice of coordinates for $\mathcal{M}$ such that the Higgs field is part of a multiplet that transforms linearly under the $O(4)$ symmetry? We now show that the answer is yes if and only if $\mathcal{M}$ has an $O(4)$ invariant fixed point. ${ }^{4}$

It is clear from the $O(4)$ transformation law eq. (2.4) for $\phi$ that the origin $\phi=0$ is an $O(4)$ invariant fixed point. Any other theory that can be formulated using fields $\phi$ which transform linearly under the $O(4)$ symmetry also must have an $O(4)$ invariant fixed point at $\phi=\mathbf{0}$. Thus, if there exists a choice of coordinates $\phi$ which transform linearly under the $O(4)$ symmetry, then the scalar manifold $\mathcal{M}$ has an $O(4)$ invariant fixed point.

Now, we prove the converse statement. Consider a general scalar manifold $\mathcal{M}$, which is described by coordinates which transform under $O(4)$ transformations and which contains an $O(4)$ invariant fixed point $P$. Is there a choice of coordinates such that the scalar fields transform linearly under the $O(4)$ symmetry? The key result we need for the proof in this direction is the linearization lemma of Coleman, Wess and Zumino [17], which states that if $P$ is an $O(4)$ invariant fixed point, there exists a set of coordinates in a neighborhood of $P$ which transform linearly under $O(4)$ transformations in some (possibly reducible) representation of $O(4)$. If this $O(4)$ representation contains the four-dimensional vector representation of $O(4)$, then the four coordinates $\phi^{i}, i=1,2,3,4$, which transform as a vector, can be combined into a Higgs doublet $H$, as in eq. (2.2). Thus, the Higgs field is part of a linear representation $H$ if and only if there is an $O(4)$ invariant fixed point whose tangent space transforms under $O(4)$ in a representation that contains the vector representation. In most of our examples, the scalar manifold is four-dimensional, and the tangent space of $P$ automatically transforms as the vector representation, so we will omit the condition that the tangent space transforms as the vector representation.

\footnotetext{
${ }^{3} \mathrm{In}$ Cartesian coordinates, $g_{i j}^{\mathrm{SM}}(\phi)=\delta_{i j}$, and it trivially follows that the Riemann curvature tensor vanishes. Since the curvature is coordinate independent, it also vanishes in polar coordinates, even though the metric is more complicated.

${ }^{4}$ In theories without custodial symmetry, the fixed point is $\mathrm{SU}(2)_{L} \times \mathrm{U}(1)_{Y}$ invariant.
} 
The condition that $\mathcal{M}$ contains an $O(4)$ fixed point divides theories into those which can and cannot be written in a form where the Higgs boson is part of a multiplet that transforms linearly under the electroweak gauge symmetry group $\mathcal{G}_{\text {gauge }}=\mathrm{SU}(2)_{L} \times \mathrm{U}(1)_{Y}$ (or the larger global custodial symmetry group $\left.\mathcal{G}=O(4)=\mathrm{SU}(2)_{L} \times \mathrm{SU}(2)_{R}\right)$. There are theories which satisfy the condition that $\mathcal{M}$ contains an $O(4)$ invariant fixed point, but which do not have relations between the couplings of the Higgs boson and the Goldstone bosons. To understand this point better, we now introduce SMEFT and HEFT.

\subsection{SMEFT}

SMEFT is an effective theory with the most general Lagrangian written in terms of SM fields, including all independent higher dimension operators with dimension greater than four, suppressed by an EFT power counting scale $\Lambda$. The independent operators at dimension six, and their renormalization [18, 19], has been worked out in detail [20-27].

In SMEFT, all operators involving scalar fields are written in terms of the Higgs doublet field $H$. For simplicity, at present we assume that the custodial symmetry group of SMEFT is $\mathcal{G}=O(4)$. The SMEFT scalar kinetic energy term, which consists of all operators built out of Higgs doublet fields with two derivatives, is

$$
\begin{aligned}
L_{\mathrm{KE}} & =\partial_{\mu} H^{\dagger} \partial^{\mu} H+\frac{1}{\Lambda^{d-4}} \sum_{i} C_{i} O_{i}^{(d)} \\
& =\partial_{\mu} H^{\dagger} \partial^{\mu} H+\frac{1}{\Lambda^{2}} C_{H D}\left(H^{\dagger} \partial_{\mu} H\right)^{*}\left(H^{\dagger} \partial^{\mu} H\right)+\cdots
\end{aligned}
$$

where the sum in the first line is over all independent mass dimension $d$ operators built out of two derivatives and Higgs doublet fields $H^{\dagger}$ and $H$, and the second line gives the explicit expression including the leading $d=6$ operator. Using eq. (2.2) to write the Higgs doublet $H$ in terms of four real scalars $\phi$, yields a scalar kinetic energy term of the form

$$
L_{\mathrm{KE}}=\frac{1}{2}\left[A\left(\frac{\phi \cdot \phi}{\Lambda^{2}}\right) \partial_{\mu} \phi \cdot \partial^{\mu} \phi+B\left(\frac{\phi \cdot \phi}{\Lambda^{2}}\right) \frac{\left(\phi \cdot \partial_{\mu} \phi\right)^{2}}{\Lambda^{2}}\right],
$$

where the arbitrary functions $A(z)$ and $B(z)$ are defined by power series expansions in their argument $z \equiv \phi \cdot \phi / \Lambda^{2}$. In the $\Lambda \rightarrow \infty$ limit, the kinetic energy term of SMEFT reduces to the SM kinetic energy term, so the function $A(z)$ satisfies $A(0)=1$. Comparison of eq. (2.21) with eq. (2.10) yields the SMEFT scalar metric

$$
g_{i j}(\phi)=A\left(\frac{\phi \cdot \phi}{\Lambda^{2}}\right) \delta_{i j}+B\left(\frac{\phi \cdot \phi}{\Lambda^{2}}\right) \frac{\phi_{i} \phi_{j}}{\Lambda^{2}}
$$

The Riemann curvature tensor $R_{i j k l}(\phi)$ of the curved scalar manifold $\mathcal{M}$ in SMEFT can be calculated from the above metric. The SM is a special case of the SMEFT in which all higher dimension operators with $d>4$ are set to zero, or equivalently, one takes the limit $\Lambda \rightarrow \infty$. From eq. (2.22), we see that in this limit the SMEFT metric yields the SM scalar metric $g_{i j}^{\mathrm{SM}}(\phi)=\delta_{i j}$ in Cartesian coordinates, and $\mathcal{M} \rightarrow \mathbb{R}^{4}$ becomes flat with vanishing Riemann curvature tensor. 
Most composite Higgs models [28, 29] can be written in SMEFT form. A simple example is the $\mathrm{SO}(5) \rightarrow \mathrm{SO}(4)$ composite Higgs model [30]. The symmetry breaking field lives on a sphere of radius $f$ in five dimensions, and can be written as

$$
\left[\begin{array}{c}
\phi \\
\sqrt{f^{2}-\phi \cdot \phi}
\end{array}\right]
$$

$\phi$ is the SMEFT field, and the Lagrangian can be written in SMEFT form. In general, composite Higgs theories solve the hierarchy problem by vacuum misalignment. There is a field configuration where the vacuum is "aligned," so that the electroweak symmetry is unbroken. This is the point $\phi=\mathbf{0}$ of SMEFT, and $\phi$ measures deviations from this point, as in eq. (2.23). In the neighborhood of $\boldsymbol{\phi}=\mathbf{0}, \boldsymbol{\phi}$ gives a linear representation of $O(4)$. For HEFT to reduce to SMEFT form, this representation must transform as the vector of $O(4)$. Composite Higgs models which are consistent with experimental data are of this type $[31,32]$.

The SMEFT is the EFT generalization of the SM where the scalar manifold has an $O(4)$ invariant fixed point, so that the Lagrangian can be written in terms of the Higgs doublet field $H$ or the four-dimensional vector field $\phi$ on which the $O(4)$ symmetry acts linearly. This restriction is not enough to give the same scattering amplitudes of Higgs bosons and Goldstone bosons (longitudinal gauge bosons) as the SM, which can be verified by explicit computation using eq. (2.21). In refs. [1, 33], it was shown that the high energy behavior of the cross sections for $W_{L} W_{L} \rightarrow W_{L} W_{L}$ and $W_{L} W_{L} \rightarrow h h$ scattering depend on two sectional curvatures which can be obtained from the Riemann curvature tensor $R_{i j k l}(\phi)$. The one-loop radiative correction in the scalar sector also depends on the Riemann curvature tensor $R_{i j k l}(\phi)$ [1]. The details of these calculations are presented later in this paper. The important point is that the $\phi \phi \rightarrow \phi \phi$ scattering cross sections and the one-loop radiative correction in SMEFT are equal to the SM values if and only if $\mathcal{M}$ is flat, i.e. the Riemann curvature tensor of SMEFT vanishes. This statement is a coordinate-independent condition, which is true in the SM using either Cartesian or polar coordinates. Thus, the intuitive idea that the Goldstone boson and Higgs boson directions in figure 1 are related in the SM can be formulated precisely as the condition that $\mathcal{M}$ in the SM is a four-dimensional flat Euclidean space.

\section{$2.3 \quad$ HEFT}

HEFT is a generalization of the SM using the polar coordinate form of the SM Lagrangian, eq. (2.12). The theory is written in terms of three angular coordinates $\pi^{a} / v$ that parametrize a unit vector $\boldsymbol{n}(\pi) \in S^{3}$, and one or more coordinates $\left\{h_{i}\right\}$. As in the $\mathrm{SM}$, the unit vector $\boldsymbol{n}$ parametrizes the Goldstone bosons directions [34-38]. Here we restrict to one additional $h$ field. The case of multiple $\left\{h_{i}\right\}$ is considered in section 6 . The coordinate $h$ is chosen so that $h=0$ is the ground state. The HEFT Lagrangian is

$$
L=\frac{1}{2} v^{2} F(h)^{2}\left(\partial_{\mu} \boldsymbol{n}\right)^{2}+\frac{1}{2}\left(\partial_{\mu} h\right)^{2}-V(h)+\ldots
$$




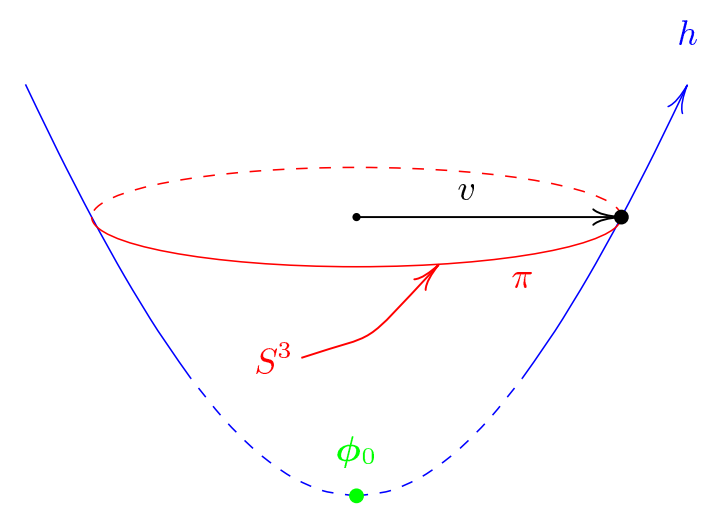

Figure 2. The HEFT scalar manifold. There is $S^{3}$ for each value of $h$. An $O(4)$ invariant fixed point exists if there is a value of $h$ for which the radius of $S^{3}$ vanishes. The fixed point $\phi_{0}$ at $h=h_{*}$ is shown in a dotted region of $\mathcal{M}$ since it need not exist. There is no boundary at the transition between the solid and dotted regions, if the dotted region does not exist. Instead, the manifold can extend to infinity, or is smoothly connected without a point where $F(h)=0$. SMEFT has a scalar manifold where $\phi=0$ is an $O(4)$ invariant fixed point that always exists, and are like the HEFT manifold including the dotted section.

where $F(h)$ is an arbitrary dimensionless function with a power series expansion in $h / v$ [39], normalized so that

$$
F(0)=1,
$$

since the radius of $S^{3}$ in the vacuum is fixed to be $v$ by the gauge boson masses. The HEFT manifold is shown schematically in figure 2. $\mathcal{M}$ has a coordinate $h$, with an $S^{3}$ fiber at each value of $h$. While $h$ is often called the radial direction by analogy with the polar coordinate form of the SM, in HEFT, $h$ is simply a scalar field, and need not be the radius of anything. HEFT power counting is discussed in [40], and is a combination of chiral power counting [41, 42] and naive dimensional analysis [43]. The terms omitted in eq. (2.24) are the NLO operators [44-48].

The $O(4)$ transformation laws for $h$ and $\boldsymbol{n}$ are given in eq. (2.13), so $h$ is invariant and $\boldsymbol{n}$ transforms non-linearly. The SM and SMEFT are both special cases of HEFT. In the $\mathrm{SM}$, the radial function is

$$
F^{\mathrm{SM}}(h)=\left(1+\frac{h}{v}\right)
$$

The SMEFT kinetic energy term eq. (2.21) yields the polar coordinate kinetic energy term

$$
L=\frac{1}{2}(v+h)^{2} A(z)\left(\partial_{\mu} \boldsymbol{n}\right)^{2}+\frac{1}{2}[A(z)+z B(z)]\left(\partial_{\mu} h\right)^{2}, \quad z=\frac{(v+h)^{2}}{\Lambda^{2}} .
$$

This kinetic energy term can be put into the standard form of HEFT by performing a field redefinition on $h$ to make the coefficient of the $\left(\partial_{\mu} h\right)^{2}$ term equal to $1 / 2$. Thus, the HEFT scalar metric for one singlet Higgs field is

$$
g_{i j}(\phi)=\left[\begin{array}{cc}
F(h)^{2} g_{a b}(\pi) & 0 \\
0 & 1
\end{array}\right] \text {, }
$$


where the function $F(h)$ is parametrized by coefficients $c_{n}, n \geq 1$,

$$
F(h)=1+c_{1}\left(\frac{h}{v}\right)+\frac{1}{2} c_{2}\left(\frac{h}{v}\right)^{2}+\cdots .
$$

The coefficient $c_{1}$ is already constrained by experiment to be equal to its SM value $c_{1}=1$ to a precision of about $10 \%$. The coefficient $c_{2}$ is not constrained at present. The HEFT scalar metric reduces to the SM scalar metric when $F(h)=F^{\mathrm{SM}}(h)=1+h / v$.

In the SMEFT, the functions $A$ and $B$ in eq. (2.22) are expanded out in powers of $\boldsymbol{\phi} \cdot \boldsymbol{\phi}$, whereas in the HEFT literature, they are treated as arbitrary (unexpanded) functions.

When is it possible to rewrite HEFT in SMEFT form? We have seen that a necessary and sufficient condition is that there must exist an $O(4)$ invariant fixed point $P$ on $\mathcal{M}$. One can then define $\phi$ as coordinates around $P$ and write the Lagrangian in terms of $\phi$. The general HEFT manifold consists of $h$ and a sequence of spheres of radius $v F(h)$ fibered over each point of $h$. The HEFT manifold is depicted in figure 2. $O(4)$ acts on the point $\boldsymbol{n}$ on the surface of $S^{3}$ by rotation, so that $O(4)$ maps points on the the red curve onto itself. No point of $S^{3}$ is invariant under the full $O(4)$ group, so the only way to have an $O(4)$ invariant fixed point is if the sphere has zero radius, i.e. if $F\left(h_{*}\right)=0$ for some $h_{*}$. Such a point may not exist; its existence depends on the structure of the HEFT manifold. For example, if $F(h)=e^{h / v} \cosh (1+h / f)$ the HEFT manifold has no $O(4)$ invariant fixed point. In the SM, $F(h)$ is given by eq. (2.26), and $F\left(h_{*}\right)=0$ at $h_{*}=-v$. If there is an $O(4)$ fixed point, the HEFT can be written as a SMEFT. Some examples are given in refs. [48-50].

To summarize, HEFT with no $O(4)$ invariant point, i.e. no point where $F(h)=0$, cannot be written in SMEFT form, and hence cannot be written using a doublet field $H$ (or equivalently, a four-dimensional vector field $\phi$ ) which transforms linearly under the electroweak gauge symmetry. This statement answers the question posed in the introduction: when do the scalar fields of HEFT transform linearly or non-linearly under the gauge symmetry? They transform linearly if and only if $F\left(h_{*}\right)=0$ for some $h_{*}$, so that there is a $O(4)$ fixed point.

Thus, we have shown that the relationship of the SM, SMEFT and HEFT is described by the hierarchy $\mathrm{SM} \subseteq \mathrm{SMEFT} \subseteq \mathrm{HEFT}$. SMEFT is a special case of HEFT when there is a value of the Higgs field $h_{*}$ where $F\left(h_{*}\right)=0$. The SM is the special case of SMEFT (and HEFT) when there are no higher dimension operators in the theory, and so $\mathcal{M}$ is flat.

One can convert the SMEFT Lagrangian to HEFT form using eq. (2.11) to switch from Cartesian to polar coordinates. One can attempt to convert from HEFT to SMEFT form using

$$
\frac{\phi}{(\phi \cdot \phi)^{1 / 2}}=n
$$

with $(\phi \cdot \phi)^{1 / 2}$ some function of $h$. This substitution gives a Lagrangian $L(\phi)$ that need not be analytic in $\phi$. However, if there is an $O(4)$ fixed point, then there is a suitable change of variables such that the resulting Lagrangian is analytic in $\phi$. 
Scattering amplitudes are evaluated in perturbation theory by expanding the action in small fluctuations about the vacuum (the black dot) in figure 2. The curvature of $\mathcal{M}$ is a local quantity, given by the metric and its derivatives up to second order, evaluated at the vacuum state. Scattering amplitudes, and hence experimentally measurable cross sections depend directly on the curvature $[1,33]$, so the curvature of the EFT scalar manifold can be determined experimentally.

Whether there is an $O(4)$ invariant fixed point where $F\left(h_{*}\right)=0$ is a non-perturbative question, since $F(0)=1$ in the ground state. One has to move a distance of at least $h \sim v$ away from the ground state to probe the existence of a fixed point where $F(h)$ vanishes.

\section{Covariant formalism for curved scalar field space}

In this section, we review the well-known geometric formulation of non-linear sigma models $[3,4,11,42,51]$. The use of functional methods for quantum corrections, combined with a covariant formalism sheds light on a number of technical issues identified in refs. [37, 52]. This covariant formalism has wide applicability - the CCWZ phenomenological Lagrangian is a special case of the geometric approach in a particular choice of coordinates, as discussed in section 4 .

\subsection{Scalar fields on a curved manifold $\mathcal{M}$}

Consider $N$ real scalar fields $\phi^{i}$ which are the coordinates of a curved scalar manifold $\mathcal{M}$. The scalar action for the $O\left(p^{2}\right)$ Lagrangian (with no gauge fields) containing all operators with up to two derivatives is

$$
S=\int d^{4} x \mathcal{L}[\phi(x)]=\int d^{4} x\left(\frac{1}{2} g_{i j}(\phi)\left(\partial_{\mu} \phi\right)^{i}\left(\partial^{\mu} \phi\right)^{j}+\mathcal{I}(\phi)\right),
$$

where $\mathcal{I}(\phi)$ is an invariant scalar density on $\mathcal{M}$. The two-derivative terms define the scalar metric $g_{i j}(\phi)$ of $\mathcal{M}$. Under a scalar field redefinition or change of scalar coordinates $\phi^{\prime}(\phi)$, the derivative $\left(\partial_{\mu} \phi^{i}\right)$ transforms as a contravariant vector

$$
\partial_{\mu} \phi^{i}=\left(\frac{\partial \phi^{\prime i}}{\partial \phi^{j}}\right) \partial_{\mu} \phi^{j}
$$

and the metric $g_{i j}(\phi)$ transforms as a tensor with two lower indices,

$$
g_{i j}^{\prime}=\left(\frac{\partial \phi^{k}}{\partial \phi^{\prime i}}\right)\left(\frac{\partial \phi^{l}}{\partial \phi^{\prime j}}\right) g_{k l} .
$$

Thus, the Lagrangian also is an invariant scalar density. The potential $\mathcal{I}(\phi)$ is non-zero, in general. It is a constant if all the fields $\phi^{i}$ are exact Goldstone bosons of an enlarged global symmetry.

The first variation of the action yields the equation of motion for the field $\phi$. Under an infinitesimal variation $\phi \rightarrow \phi+\eta$, the linear in $\eta$ variation of the action is

$$
\delta S=\int d^{4} x\left(-g_{i j}\left(\mathcal{D}_{\mu}\left(\partial^{\mu} \phi\right)\right)^{i}+\mathcal{I}_{, j}\right) \eta^{j}
$$


where

$$
\left(\mathcal{D}_{\mu} \eta\right)^{i} \equiv \partial_{\mu} \eta^{i}+\Gamma_{k j}^{i}\left(\partial_{\mu} \phi\right)^{k} \eta^{j}
$$

is the covariant derivative on a vector field $\eta^{i}$ and $\Gamma_{j k}^{i}(\phi)$ is the Christoffel symbol. From eq. (3.4), one obtains the classical equation of motion

$$
E_{j}=g_{i j}\left(\mathcal{D}_{\mu}\left(\partial^{\mu} \phi\right)\right)^{i}-\mathcal{I}_{, j}=g_{i j}\left(\partial^{2} \phi^{i}+\Gamma_{k j}^{i}\left(\partial_{\mu} \phi\right)^{k}\left(\partial_{\mu} \phi\right)^{j}\right)-\mathcal{I}_{, j}=0,
$$

which is the wave equation for $\phi$ on the curved manifold $\mathcal{M}$.

The second variation of the action under an infinitesimal variation $\phi \rightarrow \phi+\eta$ is

$$
\delta^{2} S=\frac{1}{2} \int d^{4} x\left[g_{i j}\left(\mathcal{D}_{\mu} \eta\right)^{i}\left(\mathcal{D}_{\mu} \eta\right)^{j}-R_{i j k l} \eta^{i}\left(\partial_{\mu} \phi\right)^{j} \eta^{k}\left(\partial_{\mu} \phi\right)^{l}-E_{j} \Gamma_{k l}^{j} \eta^{k} \eta^{l}+\mathcal{I}_{; i j} \eta^{i} \eta^{j}\right],
$$

where $R_{i j k l}$ is the Riemann curvature tensor and

$$
\mathcal{I}_{; i j}=\nabla_{i} \nabla_{j} \mathcal{I}=\frac{\partial^{2} \mathcal{I}}{\partial \phi^{i} \partial \phi^{j}}-\Gamma_{i j}^{k} \frac{\partial \mathcal{I}}{\partial \phi^{k}} .
$$

Eq. (3.7) is not covariant because of the third term which depends explicitly on the connection $\Gamma_{j k}^{i}$. This term, however, vanishes on shell since it is proportional to the equation of motion $E_{i}$. The non-covariant term leads to non-covariant divergences in Green functions which vanish in $S$-matrix elements. Even though they have no physical consequences, the appearance of non-covariant terms is puzzling since the original theory is covariant. The non-covariant terms occur because the infinitesimal variation $\phi \rightarrow \phi+\eta$ is not a covariant parameterization of fluctuations to second order in $\eta$, as was explained in ref. [3, 4]. ${ }^{5}$

The variation of the scalar field $\eta^{i}=\delta \phi^{i}$ should transform as a vector under a change of coordinates. However, under a change of coordinates,

$$
\phi^{i}(\phi+\eta)=\phi^{i}(\phi)+\left(\frac{\partial \phi^{\prime i}}{\partial \phi^{j}}\right) \eta^{j}+\frac{1}{2}\left(\frac{\partial^{2} \phi^{i}}{\partial \phi^{j} \partial \phi^{k}}\right) \eta^{j} \eta^{k}+\ldots \equiv \phi^{i}(\phi)+\eta^{i}
$$

implies that

$$
\eta^{i}=\left(\frac{\partial \phi^{\prime i}}{\partial \phi^{j}}\right) \eta^{j}+\frac{1}{2}\left(\frac{\partial^{2} \phi^{\prime i}}{\partial \phi^{j} \partial \phi^{k}}\right) \eta^{j} \eta^{k}+\ldots,
$$

which is the correct transformation law for a vector at first order in $\eta$, but not at second order. The solution to this problem is to use geodesic coordinates to parametrize fluctuations in $\phi$, as shown in ref. [4]. The equation for a geodesic on $\mathcal{M}$ parameterized by $\lambda$ is

$$
\frac{\mathrm{d}^{2} \phi^{i}}{\mathrm{~d} \lambda^{2}}+\Gamma_{j k}^{i}(\phi) \frac{\mathrm{d} \phi^{j}}{\mathrm{~d} \lambda} \frac{\mathrm{d} \phi^{k}}{\mathrm{~d} \lambda}=0 .
$$

Solving this equation in perturbation theory, starting at $\phi^{i}=\phi_{0}^{i}$ with tangent vector $\eta^{i}$ gives

$$
\phi^{i}=\phi_{0}^{i}+\lambda \eta^{i}-\frac{1}{2} \lambda^{2} \Gamma_{j k}^{i}\left(\phi_{0}\right) \eta^{j} \eta^{k}+\ldots
$$

\footnotetext{
${ }^{5}$ An explicit calculation of the $O(N)$ model in the linear and non-linear formulations, and a computation of the non-covariant terms can be found in $\mathrm{v} 1$ of this paper on arXiv.org.
} 
Fluctuations in $\phi$ are parameterized by picking $\eta^{i}$ to be tangent vector such that the geodesic reaches $\phi+\delta \phi$ at $\lambda=1$, i.e. using the variation

$$
\phi^{i} \rightarrow \phi^{i}+\eta^{i}-\frac{1}{2} \Gamma_{j k}^{i} \eta^{j} \eta^{k}+\mathcal{O}\left(\eta^{3}\right)
$$

which suffices to restore the correct transformation law for the vector $\eta^{\prime}$ to second order in the expansion,

$$
\eta^{\prime i}=\left(\frac{\partial \phi^{\prime i}}{\partial \phi^{j}}\right) \eta^{j}
$$

Expanding the action in the geodesic fluctuation $\eta$ to quadratic order in $\eta$ yields

$$
S[\phi+\eta]=S[\phi]+\frac{\delta S}{\delta \phi^{i}}\left(\eta^{i}-\frac{1}{2} \Gamma_{j k}^{i} \eta^{j} \eta^{k}\right)+\frac{\delta^{2} S}{\delta \phi^{i} \delta \phi^{j}} \eta^{i} \eta^{j}+\mathcal{O}\left(\eta^{3}\right)
$$

which shows that there is a quadratic in $\eta$ term proportional to the equation of motion operator $E_{i} \equiv\left(\delta S / \delta \phi^{i}\right)$. This contribution exactly cancels the non-covariant term of eq. (3.7), yielding a second variation of the action which transforms covariantly

$$
\delta^{2} S=\frac{1}{2} \int d^{4} x\left[g_{i j}\left(\mathcal{D}_{\mu} \eta\right)^{i}\left(\mathcal{D}^{\mu} \eta\right)^{j}-R_{i j k l} \eta^{i}\left(\partial_{\mu} \phi\right)^{j} \eta^{k}\left(\partial^{\mu} \phi\right)^{l}+\left(\nabla_{i} \nabla_{j} \mathcal{I}\right) \eta^{i} \eta^{j}\right] .
$$

An equivalent way to implement the covariant expansion is to promote ordinary functional derivatives to covariant functional derivatives [11],

$$
\nabla_{i} S=\frac{\delta S}{\delta \phi^{i}}, \quad \nabla_{i} \nabla_{j} S=\frac{\delta^{2} S}{\delta \phi^{i} \delta \phi^{j}}-\Gamma_{i j}^{k} \frac{\delta S}{\delta \phi^{k}} .
$$

The second variation of the action enters the one-loop correction to the functional integral,

$$
\Gamma_{\text {one-loop }}=\frac{i}{2} \log \operatorname{det}\left(-g^{i k} \frac{\delta^{2} S}{\delta \eta^{k} \delta \eta^{j}}\right)
$$

The one-loop corrections computed using eq. (3.16) are covariant, since $\delta^{2} S$ is covariant. The two forms for $\delta^{2} S$, eq. (3.7) and eq. (3.16), differ in the form for $\phi^{\prime}$, eq. (3.10) and eq. (3.13), i.e. by a field redefinition. Thus the two formulations have the same $S$-matrix, but different Green functions. The covariant form eq. (3.16) has covariant Green functions and $S$-matrix elements, so the non-covariant version eq. (3.7) has covariant $S$-matrix elements (since they are not changed by field redefinitions) but non-covariant Green functions.

The one-loop radiative correction can be computed from eq. (3.18). For renormalization of the theory at one-loop in dimensional reqularization, we only require the divergent one-loop contribution to the Lagrangian. This contribution can be extracted using the covariant derivative formalism in refs. [11,53-56], which gives the same result as an earlier explicit computation by 't Hooft [57]. The results are given in eq. (3.45), after we have discussed the gauged version of eq. (3.18). Since $\delta^{2} S$ is covariant, the radiative corrections are also covariant when computed this way. 


\subsection{Global symmetry on $\mathcal{M}$}

We now consider the global symmetries of the ungauged action eq. (3.1). The global symmetries of the scalar kinetic energy term are the isometries of $\mathcal{M}$. These isometries are specified by a set of vector fields $t_{\alpha}^{i}$, the Killing vectors of $\mathcal{M}$, where the different isometries are labelled by $\alpha$. The Killing vectors generate the infinitesimal field transformations

$$
\delta_{\theta} \phi^{i}=\theta^{\alpha} t_{\alpha}^{i}(\phi),
$$

where $\theta^{\alpha}$ are infinitesimal parameters. The gradient of $\phi$ transforms as

$$
\delta_{\theta}\left(\partial_{\mu} \phi^{i}\right)=\theta^{\alpha}\left(\frac{\partial t_{\alpha}^{i}}{\partial \phi^{j}}\right)\left(\partial_{\mu} \phi^{j}\right)
$$

For the $O(N)$ sigma model, the global symmetries of the scalar kinetic energy term are $\mathcal{G}=O(N)$ transformations on the $N$-component real scalar field $\phi$. The $N(N-1) / 2$ Killing vectors of $\mathcal{M}$ are

$$
t_{a b}^{i}(\phi)=i\left[M_{a b}\right]_{j}^{i} \phi^{j}=i\left(M_{a b} \phi\right)^{i}
$$

where $M_{a b}, 1 \leq a<b \leq N$, are the $N \times N$ anti-symmetric Hermitian matrices

$$
\left[M_{a b}\right]_{j}^{i}=-i\left(\delta_{a}^{i} \delta_{j b}-\delta_{j a} \delta_{b}^{i}\right), \quad 1 \leq a<b \leq N,
$$

and the label $\alpha$ has been replaced by the bi-index $a b$. The Killing vectors in eq. (3.21) are linear in the $N$ Cartesian components of the field $\phi$, but not in the $N$ polar components. The $O(N)$ Killing vectors can be divided into the $(N-1)(N-2) / 2$ Killing vectors of the unbroken subgroup $\mathcal{H}=O(N-1)$ and the $(N-1)$ Killing vectors which are spontaneously broken,

$$
\begin{array}{ccc}
t_{a b}^{i}(\phi) & =i\left(M_{a b} \phi\right)^{i}=\left(\delta^{i}{ }_{a} \phi_{b}-\delta^{i}{ }_{b} \phi_{a}\right), & 1 \leq a<b<N \\
t_{a N}^{i}(\phi) & =i\left(M_{a N} \phi\right)^{i}=\left(\delta^{i}{ }_{a} \phi_{N}-\delta^{i}{ }_{N} \phi_{a}\right), & 1 \leq a<N .
\end{array}
$$

Restricting to the scalar submanifold $S^{N-1}$ such that $\langle\phi \cdot \phi\rangle=v^{2}$ with $h=0$, yields $N_{\varphi}=(N-1)$ independent real scalar fields $\varphi^{a}$. The Killing vectors of $S^{N-1}$ on the first line of eq. (3.23) act linearly on the $\varphi^{a}$ in both Cartesian and polar coordinates. Those on the second line act non-linearly, since $\phi^{N}=\sqrt{v^{2}-\varphi \cdot \varphi}$. Explicitly,

$$
\begin{aligned}
t_{a b}^{i}(\varphi) & =i\left(M_{a b} \varphi\right)^{i}=\left(\delta^{i}{ }_{a} \varphi_{b}-\delta^{i}{ }_{b} \varphi_{a}\right), & & 1 \leq a<b<N, \\
t_{a N}^{i}(\varphi) & =i\left(M_{a N} \phi\right)^{i}=\delta^{i}{ }_{a} \sqrt{v^{2}-\varphi \cdot \varphi}, & & 1 \leq a<N,
\end{aligned}
$$

for $i=1, \ldots, N-1$.

The infinitesimal field transformations generated by the Killing vectors in eq. (3.19) leave the action eq. (3.1) invariant, provided that

$$
\mathscr{L}_{t_{\alpha}} g=0, \quad \mathscr{L}_{t_{\alpha}} \mathcal{I}=0,
$$


where $\mathscr{L}_{t_{\alpha}}$ is the Lie derivative for Killing vector $t_{\alpha}^{i}$. The first condition in eq. (3.25) is the definition of a Killing vector; it is an isometry of the metric. The second condition is that the potential is invariant.

The Lie bracket $\left[t_{\alpha}, t_{\beta}\right]$ of two isometries is also an isometry since

$$
\left[\mathscr{L}_{t_{\alpha}}, \mathscr{L}_{t_{\beta}}\right]=\mathscr{L}_{\left[t_{\alpha}, t_{\beta}\right]},
$$

so the Killing vectors form the symmetry algebra

$$
\left[t_{\alpha}, t_{\beta}\right]^{i}=f_{\alpha \beta}^{\gamma} t_{\gamma}^{i}
$$

Evaluating the Lie bracket gives

$$
\left[t_{\alpha}, t_{\beta}\right]^{i}=t_{\alpha}^{k} t_{\beta, k}^{i}-t_{\beta}^{k} t_{\alpha, k}^{i}=f_{\alpha \beta}^{\gamma} t_{\gamma}^{i} .
$$

Note that the above equation also holds with the ordinary derivative $t_{\alpha, k}^{i}$ replaced by the covariant derivative

$$
t_{\alpha ; k}^{i}=t_{\alpha, k}^{i}+\Gamma_{k j}^{i} t_{\alpha}^{j}=\frac{\partial t_{\alpha}^{i}}{\partial \phi^{k}}+\Gamma_{k j}^{i} t_{\alpha}^{j},
$$

since the Christoffel symbol is symmetric in lower indices, and cancels in the antisymmetric derivative of eq. (3.28). The Killing vectors in eq. (3.24) are a non-trivial example of Killing vectors which form a closed set under the Lie bracket.

As noted at the beginning of the section, a covariant treatment guarantees that vectors $\eta^{i}$ transform the same way as $\partial_{\mu} \phi^{i}$ under isometries, e.g.

$$
\delta_{\theta} \eta^{i}=\theta^{\alpha}\left(\frac{\partial t_{\alpha}^{i}}{\partial \phi^{j}}\right) \eta^{j}
$$

which is a linear transformation law.

\subsection{Local symmetry on $\mathcal{M}$}

The global symmetries eq. (3.19) can be promoted to local symmetries by replacing the global symmetry parameters $\theta^{\alpha}$ by functions of spacetime $\theta^{\alpha}(x)$,

$$
\delta_{\theta} \phi^{i}(x)=\theta^{\alpha}(x) t_{\alpha}^{i}(\phi(x)),
$$

and introducing gauge fields.

The gauge covariant derivative of the scalar field on the curved manifold $\mathcal{M}$ is defined by

$$
\left(D_{\mu} \phi(x)\right)^{i} \equiv \partial_{\mu} \phi^{i}(x)+A_{\mu}^{\beta}(x) t_{\beta}^{i}(\phi(x))
$$

where $A_{\mu}^{\beta}(x)$ is the gauge field associated with the Killing vector $t_{\beta}^{i}(\phi)$, and the gauge coupling constant and a factor of $i$ has been absorbed into the gauge field. The gauge 
covariant derivative of the scalar field should transform the same way as $\partial_{\mu} \phi^{i}$ in eq. (3.20) under the local symmetry, which implies the transformation rule

$$
\delta_{\theta}\left(D_{\mu} \phi\right)^{i}=\theta^{\alpha}(x)\left(\frac{\partial t_{\alpha}^{i}}{\partial \phi^{j}}\right)\left(D_{\mu} \phi\right)^{j} .
$$

Consequently, the transformation law of $A_{\mu}^{\beta}(x)$ under the local symmetry is

$$
\left(\delta_{\theta} A_{\mu}^{\beta}\right) t_{\beta}^{i}=-\left(\partial_{\mu} \theta^{\beta}\right) t_{\beta}^{i}+\theta^{\beta} A_{\mu}^{\gamma}\left(t_{\gamma}^{j} \frac{\partial t_{\beta}^{i}}{\partial \phi^{j}}-t_{\beta}^{j} \frac{\partial t_{\gamma}^{i}}{\partial \phi^{j}}\right) .
$$

Using the definition of the Lie bracket in eq. (3.28), this equation yields the usual transformation law for the gauge field

$$
\delta_{\theta} A_{\mu}^{\alpha}=-\partial_{\mu} \theta^{\alpha}-f_{\beta \gamma}{ }^{\alpha} \theta^{\beta} A_{\mu}^{\gamma} .
$$

The gauged version of the Lagrangian eq. (3.1) is

$$
\mathcal{L}=\frac{1}{2} g_{i j}(\phi)\left(D_{\mu} \phi\right)^{i}\left(D^{\mu} \phi\right)^{j}+\mathcal{I}(\phi),
$$

where the partial derivatives of the scalar field have been replaced by gauge covariant derivatives eq. (3.32). The first variation of the Lagrangian gives the gauged generalization of the equation of motion eq. (3.6),

$$
\begin{aligned}
E_{i} & =g_{i j}\left(\partial_{\mu} \delta_{k}^{j}+A_{\mu}^{\beta} t_{\beta, k}^{j}\right)\left(D^{\mu} \phi\right)^{k}+g_{i l} \Gamma_{j k}^{l}\left(D^{\mu} \phi\right)^{j}\left(D_{\mu} \phi\right)^{k}-\mathcal{I}_{, i} \\
& \equiv g_{i j}\left(\mathscr{D}_{\mu}\left(D^{\mu} \phi\right)\right)^{j}-\mathcal{I}_{, i} .
\end{aligned}
$$

The gauge covariant derivative $D_{\mu} \phi$ of coordinates $\phi^{i}$ is given in eq. (3.32), and the gauged covariant derivative $\mathscr{D}_{\mu}$ on a vector field $\eta^{i}$ is

$$
\left(\mathscr{D}_{\mu} \eta\right)^{i}=\left(\partial_{\mu} \eta^{i}+\Gamma_{k j}^{i} \partial_{\mu} \phi^{k} \eta^{j}\right)+A_{\mu}^{\beta}\left(t_{\beta, j}^{i}+\Gamma_{j k}^{i} t_{\beta}^{k}\right) \eta^{j}
$$

which is the gauged generalization of eq. (3.5). Eq. (3.38) is the appropriate definition for covariant derivatives acting on vector fields. It arises in our calculation by a direct calculation to obtain the equations of motion eq. (3.37) by varying the Lagrangian eq. (3.36). One can show that eq. (3.38) transforms as

$$
\delta_{\theta}\left(\mathscr{D}_{\mu} \eta\right)^{i}=\theta^{\alpha}(x)\left(\frac{\partial t_{\alpha}^{i}}{\partial \phi^{j}}\right)\left(\mathscr{D}_{\mu} \eta\right)^{j}
$$

The derivation of eq. (3.39) relies on two useful identities. The first is obtained by differentiating eq. (3.28),

$$
f_{\alpha \beta}^{\gamma}\left(\frac{\partial t_{\gamma}^{i}}{\partial \phi^{k}}\right)=\left[\left(\frac{\partial^{2} t_{\beta}^{i}}{\partial \phi^{j} \partial \phi^{k}}\right) t_{\alpha}^{j}-\left(\frac{\partial^{2} t_{\alpha}^{i}}{\partial \phi^{j} \partial \phi^{k}}\right) t_{\beta}^{j}\right]+\left[\left(\frac{\partial t_{\beta}^{i}}{\partial \phi^{j}}\right)\left(\frac{\partial t_{\alpha}^{j}}{\partial \phi^{k}}\right)-\left(\frac{\partial t_{\alpha}^{i}}{\partial \phi^{j}}\right)\left(\frac{\partial t_{\beta}^{j}}{\partial \phi^{k}}\right)\right] .
$$


The second relation is that the Lie derivative of the Levi-Civita connection vanishes because $t_{\alpha}$ is a Killing vector. The explicit formula is

$$
0=\mathcal{L}_{t_{\alpha}} \Gamma_{k j}^{i}=t_{\alpha}^{\ell} \frac{\partial \Gamma_{k j}^{i}}{\partial \phi^{\ell}}+\frac{\partial t_{\alpha}^{\ell}}{\partial \phi^{k}} \Gamma_{\ell j}^{i}+\frac{\partial t_{\alpha}^{\ell}}{\partial \phi^{j}} \Gamma_{k \ell}^{i}-\frac{\partial t_{\alpha}^{i}}{\partial \phi^{\ell}} \Gamma_{k j}^{\ell}+\frac{\partial^{2} t_{\alpha}^{i}}{\partial \phi^{k} \partial \phi^{j}} .
$$

The first and second variations of the gauged action up to second order give the gauged versions of eqs. (3.4) and (3.16),

$$
\begin{aligned}
\delta S & =\int d^{4} x\left[-g_{i j}\left(\mathscr{D}_{\mu}\left(D_{\mu} \phi\right)\right)^{i} \eta^{j}+\mathcal{I}_{, i} \eta^{i}\right], \\
\delta^{2} S & =\frac{1}{2} \int d^{4} x\left[g_{i j}\left(\mathscr{D}_{\mu} \eta\right)^{i}\left(\mathscr{D}^{\mu} \eta\right)^{j}-R_{i j k l}\left(D_{\mu} \phi\right)^{j}\left(D^{\mu} \phi\right)^{l} \eta^{i} \eta^{k}+\left(\nabla_{i} \nabla_{j} \mathcal{I}\right) \eta^{i} \eta^{j}\right] .
\end{aligned}
$$

The gauge field now appears implicitly in every term except for those involving the potential $\mathcal{I}$. The second variation $\delta^{2} S$ depends on the curvature $R_{i j k l}$ of $\mathcal{M}$, but it does not have a term that depends on the gauge curvature (i.e. field-strength) $F_{\mu \nu}$.

The divergent one-loop contribution in $4-2 \epsilon$ dimensions for quadratic actions such as eq. (3.16) was derived by 't Hooft in ref. [57],

$$
\Delta \mathcal{L}^{1-\text { loop }}=\frac{1}{32 \pi^{2} \epsilon}\left(\frac{1}{12} \operatorname{Tr}\left[Y_{\mu \nu} Y^{\mu \nu}\right]+\frac{1}{2} \operatorname{Tr}\left[X^{2}\right]\right)
$$

where

$$
\left[Y_{\mu \nu}\right]_{j}^{i} \equiv\left[\mathscr{D}_{\mu}, \mathscr{D}_{\nu}\right]_{j}^{i}, \quad[X]_{k}^{i} \equiv-R_{j k l}^{i}\left(D_{\mu} \phi\right)^{j}\left(D^{\mu} \phi\right)^{l}+g^{i j} \mathcal{I}_{; j k} .
$$

't Hooft's original derivation is valid when the scalar metric is $\delta_{i j}$. Our form eq. (3.43) with $Y_{\mu \nu}$ and $X$ given by eq. (3.44) applies for any metric $g_{i j}$. Eq. (3.44) is the second SeeleyDeWitt coefficient in the heat-kernel expansion, and has been studied by many authors (see ref. [58] for a review).

The matrix $X$ is the mass squared term for the fluctuations $\eta$ in eq. (3.42), and $Y_{\mu \nu}$ is a field strength tensor constructed from the covariant derivative $\mathscr{D}$. An explicit computation using the identities (3.40) and (3.41) shows that $Y_{\mu \nu}$ is equal to the sum of the curvature of $\mathcal{M}$ and the curvature of the gauge field,

$$
\left[Y_{\mu \nu}\right]_{j}^{i}=\left[\mathscr{D}_{\mu}, \mathscr{D}_{\nu}\right]_{j}^{i}=R_{j k l}^{i}\left(D_{\mu} \phi\right)^{k}\left(D_{\nu} \phi\right)^{l}+F_{\mu \nu}^{\alpha} t_{\alpha ; j}^{i} .
$$

For Goldstone bosons, where $\mathcal{I}$ is a constant, $X$ and $Y_{\mu \nu}$ are both proportional to two derivatives of $\phi$ times the curvature $R_{i j k l}$, i.e. they are order $\mathcal{O}\left(R p^{2}\right)$, where $R$ is a typical curvature and $p$ is a typical momentum. Thus, the one-loop correction, which is proportional to the traces of $X^{2}$ and $Y_{\mu \nu}^{2}$, is order $\mathcal{O}\left(R^{2} p^{4}\right)$, and is $\mathcal{O}\left(p^{4}\right)$ as one expects in chiral perturbation theory. The $\mathcal{O}\left(p^{4}\right)$ correction is proportional to the square of the curvature, and vanishes if the manifold is flat, i.e. in a theory such as the SM. Thus, the $\mathrm{SM}$ is renormalizable even in non-linear coordinates; one-loop graphs do not require four-derivative counterterms. The $F_{\mu \nu}$ term in $Y_{\mu \nu}$ gives the Goldstone boson contribution to the gauge coupling $\beta$-function of order $\mathcal{O}\left(F_{\mu \nu}^{2}\right)$, and the running of operators involving field strengths of order $\mathcal{O}\left(R F_{\mu \nu} p^{2}\right)$. 
The quadratic invariants that enter eq. (3.43) are

$$
\operatorname{Tr}\left[X^{2}\right]=\left(\nabla^{i} \nabla_{j} \mathcal{I}\right)\left(\nabla^{j} \nabla_{i} \mathcal{I}\right)+R_{\left(d_{\mu} \phi\right) j\left(d_{\mu} \phi\right)}^{i} R_{\left(d_{\nu} \phi\right) i\left(d_{\nu} \phi\right)}^{j}-2\left(\nabla^{i} \nabla^{j} \mathcal{I}\right) R_{i\left(d_{\mu} \phi\right) j\left(d_{\mu} \phi\right)},
$$

and

$$
\operatorname{Tr}\left[Y_{\mu \nu} Y^{\mu \nu}\right]=R_{j\left(d_{\mu} \phi\right)\left(d_{\nu} \phi\right)}^{i} R^{j}{ }_{i\left(d_{\mu} \phi\right)\left(d_{\nu} \phi\right)}+2 R^{j}{ }_{i\left(d_{\mu} \phi\right)\left(d_{\nu} \phi\right)} F_{\mu \nu}^{\alpha}\left(t_{\alpha}^{i}\right)_{; j}+F_{\mu \nu}^{\alpha} F_{\mu \nu}^{\beta}\left(t_{\alpha}^{i}\right)_{; j}\left(t_{\beta}^{j}\right)_{; i} .
$$

Eq. (3.47) is universal and applies to many theories. The one-loop correction in HEFT, which is complicated, and was given previously in refs. $[1,16]$, is simply an expansion of eq. (3.47) into component fields. More details about the expansion are given in appendix B. As explained in ref. [1], the same formula eq. (3.47) applies to HEFT, the SM scalar sector, dilaton theories, and chiral perturbation theory.

To close this section, we consider spontaneous symmetry breaking in a theory with an invariant potential $\mathscr{L}_{t_{\alpha}} \mathcal{I}=0$, so that we have exact Goldstone bosons. The fields $\phi^{i}$ have vacuum expectation values $\left\langle\phi^{i}\right\rangle$, and transform as $\delta \phi^{i}=\theta^{\alpha} t_{\alpha}^{i}(\langle\phi\rangle)$. Thus, broken symmetries $t_{A}^{i}$ satisfy $t_{A}^{i}(\langle\phi\rangle) \neq 0$, and $t_{A}^{i}(\langle\phi\rangle)$ is a vector in the Goldstone boson direction - i.e. motion along the vector field $t_{A}^{i}$ (for each broken generator) is motion between different vacuum states with the same value of the potential $\mathcal{I}$.

In the gauged case, the Goldstone bosons are eaten, giving a mass term for the gauge bosons. The Lagrangian of eq. (3.36) gives the gauge boson mass term

$$
\mathcal{L}=\frac{1}{2} M_{B C}^{2} A_{\mu}^{B} A^{C^{\mu}}, \quad M_{B C}^{2}(\langle\phi\rangle) \equiv g_{i j}(\langle\phi\rangle) t_{B}^{i}(\langle\phi\rangle) t_{C}^{j}(\langle\phi\rangle) .
$$

The rank of $M_{B C}^{2}$ determines the number of massive gauge bosons, which cannot exceed the dimension of the manifold $\mathcal{M}$. If the number of isometries exceeds $\operatorname{dim} \mathcal{M}$, then there are unbroken symmetries. This is true in the $\mathcal{M}=S^{N}$ theory, where there are $N(N+1) / 2$ isometries which form the group $\mathcal{G}=O(N+1)$, and the unbroken subgroup $\mathcal{H}=O(N)$ has $N(N-1) / 2$ generators. The number of broken generators is $N$, which is equal to the dimension of $S^{N}$.

\section{CCWZ and non-compact groups}

In this section, we connect the geometric formalism with the explicit formulæ of CCWZ [17, 59] for Goldstone boson Lagrangians with symmetry breaking pattern $\mathcal{G} \rightarrow \mathcal{H}$. We are interested in applying the formalism to non-compact groups, and to sigma models with non-trivial metrics on $\mathcal{G} / \mathcal{H}$. Our presentation thus parallels the discussion in the original work, while pointing out differences which arise for the case of non-compact groups.

Consider a group $\mathcal{G}$ with generators $t_{\alpha}, \alpha=1, \cdots, \operatorname{dim} \mathcal{G}$, satisfying the Lie algebra $\mathfrak{g}$

$$
\left[t_{\alpha}, t_{\beta}\right]=i f_{\alpha \beta}^{\gamma} t_{\gamma}
$$

and the Jacobi identity

$$
f_{\alpha \beta}{ }^{\lambda} f_{\gamma \lambda}{ }^{\sigma}+f_{\gamma \alpha}{ }^{\lambda} f_{\beta \lambda}{ }^{\sigma}+f_{\beta \gamma}{ }^{\lambda} f_{\alpha \lambda}{ }^{\sigma}=0 .
$$


To allow for negatively curved spaces [33, 60], we do not assume that the group $\mathcal{G}$ is compact. Consequently, the Lie algebra eq. (4.1) implies that the structure constants $f_{\alpha \beta}{ }^{\gamma}$ are antisymmetric in their first two indices, $f_{\alpha \beta}^{\gamma}=-f_{\beta \alpha}{ }^{\gamma}$, but total antisymmetry of the structure constants in all three indices, which is true for compact groups, is not assumed.

The group $\mathcal{G}$ is spontaneously broken to the subgroup $\mathcal{H}$ with generators $T_{a}, a=$ $1, \cdots, \operatorname{dim} \mathcal{H}$, satisfying the Lie algebra $\mathfrak{h}$,

$$
\left[T_{a}, T_{b}\right]=i f_{a b}{ }^{c} T_{c} .
$$

The remaining broken generators of the $\operatorname{coset} \mathcal{G} / \mathcal{H}$ needed to span $\mathfrak{g}$ are given by $X_{A}$, $A=1, \cdots, \operatorname{dim} \mathcal{G} / \mathcal{H}$. The choice of the broken generators $X_{A}$ is not unique. In the familiar example of broken chiral symmetry in QCD, different choices of broken generators lead to different parameterizations of the chiral Lagrangian, e.g. by $\xi(x)$ which transforms as $\xi \rightarrow L \xi h^{\dagger}=h \xi R^{\dagger}$, or by $\mathrm{U}(x)$ which transforms as $L U R^{\dagger}[61]$.

The $\mathfrak{g}$ commutation relations of the generators $t_{\alpha}=\left\{T_{a}, X_{A}\right\}$ in eq. (4.1) decompose into the following commutation relations for the unbroken and broken generators

$$
\begin{aligned}
{\left[T_{a}, T_{b}\right] } & =i f_{a b}^{c} T_{c}, \\
{\left[T_{a}, X_{B}\right] } & =i f_{a B}{ }^{C} X_{C}+i f_{a B}{ }^{c} T_{c}, \\
{\left[X_{A}, X_{B}\right] } & =i f_{A B}{ }^{C} X_{C}+i f_{A B}^{c} T_{c} .
\end{aligned}
$$

The first line eq. (4.4a) is the Lie algebra $\mathfrak{h}$ of the subgroup $\mathcal{H}$ in eq. (4.3), which is closed under commutation, so the commutator $\left[T_{a}, T_{b}\right]$ has no term proportional to the broken generators $X_{C}$, which implies that the structure constants $f_{a b}{ }^{C}=0$.

For compact groups, complete antisymmetry of the structure constants then implies that $f_{a B}{ }^{c}=0$, so eq. (4.4b) simplifies to

$$
\left[T_{a}, X_{B}\right]=i f_{a B}^{C} X_{C},
$$

which implies that the broken generators $X_{A}$ form a (possibly reducible) representation $\mathbf{R}^{(\pi)}$ of the unbroken subgroup $\mathcal{H}$. The generators $T_{a}$ of $\mathcal{H}$ in the $\mathbf{R}^{(\pi)}$ representation are determined by the structure constants $f_{a B}{ }^{C}$,

$$
\left[T_{a}^{\mathbf{R}^{(\pi)}}\right]_{B}{ }^{C}=-i f_{a B}{ }^{C} .
$$

The $\mathfrak{h}$ commutation relations eq. (4.4a) in representation $\mathbf{R}^{(\pi)}$,

$$
\left[T_{a}^{\mathbf{R}^{(\pi)}}, T_{b}^{\mathbf{R}^{(\pi)}}\right]=i f_{a b}{ }^{c} T_{c}^{\mathbf{R}^{(\pi)}}
$$

follow from the Jacobi identity eq. (4.2).

For non-compact groups, eq. (4.5) need not be satisfied. For now, we restrict our attention to symmetry breaking patterns where eq. (4.5) holds, so $f_{a B}{ }^{c}=0$. Such cosets are called reductive cosets. Non-reductive cosets are discussed in appendix C. An example of a reductive coset is the breaking of the Lorentz group down to its rotation subgroup. For reductive cosets, the broken generators transform as a representation $\mathbf{R}^{(\pi)}$ of the unbroken 
symmetry group $\mathcal{H}$, just as in the compact case. The coset is reductive if $\mathcal{H}$ is compact, even if $\mathcal{G}$ is non-compact.

Often, there is a discrete symmetry of the Lie algebra $X_{A} \rightarrow-X_{A}$ under which the broken generators change sign. The presence of such a discrete symmetry implies that the structure constants $f_{a B}{ }^{c}$ and $f_{A B}{ }^{C}$ vanish, so the Lie algebra reduces to

$$
\begin{aligned}
{\left[T_{a}, T_{b}\right] } & =i f_{a b}{ }^{c} T_{c}, \\
{\left[T_{a}, X_{B}\right] } & =i f_{a B}{ }^{c} X_{C}, \\
{\left[X_{A}, X_{B}\right] } & =i f_{A B}{ }^{c} T_{c} .
\end{aligned}
$$

An example is chiral symmetry breaking in the strong interactions, where the broken generators are odd under parity. Cosets with such a discrete symmetry are referred to as symmetric cosets. Symmetric cosets are automatically reductive.

The CCWZ formalism picks elements of $\mathcal{G} / \mathcal{H}$ cosets using the exponential map of the broken generators $\left\{X_{A}\right\}$

$$
\xi(x) \equiv e^{i \bar{\pi} \cdot X}, \quad \bar{\pi} \cdot X \equiv \bar{\pi}^{A}(x) X_{A}=\left(\frac{\pi^{A}(x)}{F_{\pi}}\right) X_{A},
$$

where $\bar{\pi}^{A}(x)$ are the dimensionless spacetime-dependent parameters describing the Goldstone boson directions on the vacuum coset $\mathcal{G} / \mathcal{H}$. This exponential map gives a unique association between a point in the $\operatorname{coset} \mathcal{G} / \mathcal{H}$ and $\bar{\pi}^{A}(x)$ in a neighborhood of the identity element $e$. An arbitrary group element $g \in \mathcal{G}$ in the neighborhood of the identity element $e$ can be written uniquely as

$$
g=e^{i \bar{\pi} \cdot X} e^{i \alpha \cdot T}
$$

where $\alpha \cdot T \equiv \alpha^{a}(x) T_{a}$. Left action by an arbitrary group element $g \in \mathcal{G}$ on $\mathcal{G} / \mathcal{H}$ is given by

$$
T_{g}: \xi(x) \rightarrow g \xi(x)
$$

which maps a point in coset space to a new point in coset space. The transformation law for $\xi(x)$ is

$$
g \xi(x)=\xi^{\prime}(x) h(\xi(x), g), \quad g \in \mathcal{G}, h \in \mathcal{H},
$$

where $\xi^{\prime}(x)$ is a new coset and $h \in \mathcal{H}$ is an implicit function of $g \in \mathcal{G}$ and the original coset $\xi(x)$. Using the identity

$$
g \xi(x)=\left(g \xi(x) g^{-1}\right) g, \quad g \xi(x) g^{-1}=\exp \left(i \bar{\pi}(x) \cdot\left(g X g^{-1}\right)\right),
$$

one sees that if $g=h_{0} \in \mathcal{H}$ is an unbroken symmetry transformation, then $h\left(\xi(x), h_{0}\right)=h_{0}$. In addition, $\xi^{\prime}=h_{0} \xi h_{0}^{-1}$, which implies that (since the coset is assumed reductive)

$$
\bar{\pi}^{A}(x)=\left[D^{\mathbf{R}^{(\pi)}}\left(h_{0}\right)\right]_{B}^{A} \bar{\pi}^{B}(x),
$$


where $D^{\mathbf{R}^{(\pi)}}\left(h_{0}\right)$ is the $\mathcal{H}$ transformation matrix in the $\mathbf{R}^{(\pi)}$ representation. Note that for reductive cosets, if $g=h_{0} \in H$, then $h\left(\xi(x), h_{0}\right)=h_{0}$ is a constant (i.e. it does not depend on $x$ through $\xi(x))$.

The CCWZ procedure for building a $\mathcal{G}$-invariant Lagrangian is to map all fields to the origin of coset space $\xi=1$ with $\bar{\pi}(x)=0$ by left-action by $g=\xi^{-1}$, and to define covariant derivatives in terms of this map. Explicitly, one starts with

$$
\xi^{-1} D_{\mu} \xi=\xi^{-1}\left(\partial_{\mu}+i A_{\mu}^{\alpha} t_{\alpha}\right) \xi
$$

where the gauge coupling constant has been absorbed into the normalization of the gauge field $A_{\mu}^{\alpha}$. If only a subgroup $\mathcal{G}_{\text {gauge }} \subset \mathcal{G}$ is gauged, then only gauge bosons of $\mathcal{G}_{\text {gauge }}$ appear in the above equation, or equivalently, the gauge bosons corresponding to global symmetry directions are set equal to zero. In addition, different factor gauge groups in $\mathcal{G}_{\text {gauge }}$ can have distinct gauge coupling constants. Power series expansion of $\xi^{-1} D_{\mu} \xi$ shows that it can be expressed in terms of multiple commutators, so it is an element of the Lie algebra $\mathfrak{g}$ which can be decomposed in terms of unbroken and broken generators,

$$
\begin{aligned}
\xi^{-1} D_{\mu} \xi & =\left.\xi^{-1} D_{\mu} \xi\right|_{T}+\left.\xi^{-1} D_{\mu} \xi\right|_{X}=i V_{\mu}+i\left(D_{\mu} \bar{\pi}\right) \\
\left.\xi^{-1} D_{\mu} \xi\right|_{T} & \equiv i V_{\mu}=i V_{\mu}^{a} T_{a}, \\
\left.\xi^{-1} D_{\mu} \xi\right|_{X} & \equiv i\left(D_{\mu} \bar{\pi}\right)=i\left(D_{\mu} \bar{\pi}\right)^{A} X_{A} .
\end{aligned}
$$

The above equations define $V_{\mu}$ and $\left(D_{\mu} \bar{\pi}\right)$. Usually, one normalizes the generators so that $\operatorname{Tr} t_{\alpha} t_{\beta}=\delta_{\alpha \beta} / 2$, and projects out the broken and unbroken pieces of $\xi^{-1} D_{\mu} \xi$ by taking the appropriate traces. The decomposition of a vector into a linear combination of basis vectors does not require an inner product on the vector space, so eq. (4.16) is well-defined even without this normalization of generators. An orthogonal normalization of generators is not possible for non-compact $\mathcal{G}$, but eq. (4.16) is well-defined. Under an unbroken symmetry transformation $h \in \mathcal{H}, V_{\mu}$ transforms like a gauge field

$$
V_{\mu} \rightarrow h V_{\mu} h^{-1}-\left(\partial_{\mu} h\right) h^{-1}
$$

and $\left(D_{\mu} \bar{\pi}\right)$ transforms by adjoint action by $\mathcal{H}$ in the representation $\mathbf{R}^{(\pi)}$,

$$
\left(D_{\mu} \bar{\pi}\right) \rightarrow h\left(D_{\mu} \bar{\pi}\right) h^{-1}
$$

These last two equations require the reductive coset condition $f_{a B}{ }^{c}=0$. The generalization to non-reductive cosets is discussed in appendix C.

The pion covariant derivative can be decomposed into a purely pionic piece and a gauge field piece,

$$
\left(D_{\mu} \bar{\pi}\right)^{A} \equiv[e(\bar{\pi})]_{B}^{A}\left(\partial_{\mu} \bar{\pi}^{B}\right)+F_{\alpha}^{A}(\bar{\pi}) A_{\mu}^{\alpha}
$$

where $[e(\bar{\pi})]_{B}^{A}$ are vierbeins of the $\mathcal{G} / \mathcal{H}$ vacuum manifold, and $F_{\alpha}^{A}(\bar{\pi})$ are related to the Killing vectors of $\mathcal{G} / \mathcal{H}$. 
For groups where $\left(D_{\mu} \bar{\pi}\right)^{A}$ transforms as a single irreducible representation $\mathbf{R}^{(\pi)}$, as in $\mathrm{QCD}$, the simplest invariant Lagrangian is the $\mathcal{O}\left(p^{2}\right)$ term

$$
\begin{aligned}
L & =\frac{1}{2} F_{\pi}^{2} \sum_{A}\left(D_{\mu} \bar{\pi}\right)^{A}\left(D^{\mu} \bar{\pi}\right)^{A} \\
& =\frac{1}{2} F_{\pi}^{2} \sum_{A}[e(\bar{\pi})]_{B}^{A}[e(\bar{\pi})]_{C}^{A}\left(\partial_{\mu} \bar{\pi}\right)^{B}\left(\partial^{\mu} \bar{\pi}\right)^{C}+\cdots \\
& \equiv \frac{1}{2} g_{B C}(\bar{\pi})\left(\partial_{\mu} \bar{\pi}\right)^{B}\left(\partial^{\mu} \bar{\pi}\right)^{C}+\cdots
\end{aligned}
$$

where $F_{\pi}$ is the Goldstone boson decay constant, and the ellipsis denotes terms depending on the gauge fields. The Lagrangian eq. (4.20) defines the scalar field metric of the $\mathcal{G} / \mathcal{H}$ vacuum manifold,

$$
g_{B C}(\bar{\pi})=F_{\pi}^{2} \sum_{A}[e(\bar{\pi})]_{B}^{A}[e(\bar{\pi})]_{C}^{A} .
$$

If the representation is reducible, the sum in eq. (4.20) can be divided into sums over the individual irreducible representations, with arbitrary weights for each irreducible representation. The most general $\mathcal{O}\left(p^{2}\right)$ term allowed is

$$
L=\frac{1}{2} F_{\pi}^{2} \sum_{A, B} \eta_{A B}\left(D_{\mu} \bar{\pi}\right)^{A}\left(D^{\mu} \bar{\pi}\right)^{B},
$$

where $\eta_{A B}$ is a symmetric tensor invariant under the adjoint action of $\mathcal{H}$, eq. (4.18). $\eta_{A B}$ is a positive definite matrix so that the pion kinetic energies have the correct sign. Note that $\eta_{A B}$ is a constant, i.e. it does not depend on $\pi$. One can always define a positive definite kinetic energy if $\mathcal{H}$ is a compact subgroup, e.g. by choosing $\eta_{A B}=\delta_{A B}$. In summary, the most general scalar metric for $\mathcal{G} / \mathcal{H}$ is

$$
g_{C D}(\bar{\pi})=F_{\pi}^{2} \sum_{A, B} \eta_{A B}[e(\bar{\pi})]_{C}^{A}[e(\bar{\pi})]_{D}^{B},
$$

and the Killing vectors in section 3.2 are given by

$$
t_{\alpha}^{A}(\bar{\pi})=\left[e^{-1}(\bar{\pi})\right]_{B}^{A} F_{\alpha}^{B}(\bar{\pi})
$$

where $\left[e^{-1}(\bar{\pi})\right]$ is the inverse vierbein, which satisfies the identity

$$
\left[e^{-1}(\bar{\pi})\right]_{B}^{A}[e(\bar{\pi})]_{C}^{B}=\delta_{C}^{A} .
$$

Eq. (4.24) can easily be derived by looking at the shift $\bar{\pi} \rightarrow \bar{\pi}+\delta \bar{\pi}$ for an infinitesimal $\mathcal{G}$ transformation.

For the HEFT example, we need to evaluate the curvature tensors at the vacuum field configuration $\bar{\pi}^{A}=0$, which requires knowing the metric tensor to quadratic order in $\bar{\pi}$. The curvature at any other point can then be obtained using left-action by $\mathcal{G}$. Expanding 
eq. (A.4) and using the most general Lie algebra relations eqs. (4.4a), (4.4b) and (4.4c), one obtains

$$
\begin{aligned}
\left(D_{\mu} \bar{\pi}\right)^{A}= & \partial_{\mu} \bar{\pi}^{A}+\frac{1}{2} f_{C B}{ }^{A} \bar{\pi}^{C} \partial_{\mu} \bar{\pi}^{B}+\frac{1}{6} f_{D \alpha}{ }^{A} f_{C B}{ }^{\alpha} \bar{\pi}^{D} \bar{\pi}^{C} \partial_{\mu} \bar{\pi}^{B}+\ldots \\
& +A_{\mu}^{A}+f_{B \alpha}{ }^{A} \bar{\pi}^{B} A_{\mu}^{\alpha}+\frac{1}{2} f_{C \beta}{ }^{A} f_{B \alpha}{ }^{\beta} \bar{\pi}^{B} \bar{\pi}^{C} A_{\mu}^{\alpha}+\ldots
\end{aligned}
$$

The term $A_{\mu}^{A}$ only involves the broken generators, and it is the square of this term in the kinetic energy which results in the broken gauge bosons acquiring a mass proportional to $F_{\pi}^{2}$. From eq. (4.19), the vierbein is

$$
[e(\bar{\pi})]_{B}^{A}=\delta_{B}^{A}+\frac{1}{2} f_{C B}{ }^{A} \bar{\pi}^{C}+\frac{1}{6} f_{D \alpha}{ }^{A} f_{E B}{ }^{\alpha} \bar{\pi}^{D} \bar{\pi}^{E}+\ldots
$$

Using eq. (4.23), the metric $g_{C D}(\bar{\pi})$ is

$$
\begin{aligned}
\frac{1}{F_{\pi}^{2}} g_{C D}(\bar{\pi})= & \eta_{C D}+\frac{1}{2}\left(\eta_{A D} f_{E C}{ }^{A}+\eta_{C B} f_{E D}{ }^{B}\right) \bar{\pi}^{E} \\
& +\left(\frac{1}{6} \eta_{C B} f_{E \alpha}{ }^{B} f_{F D}{ }^{\alpha}+\frac{1}{6} \eta_{A D} f_{E \alpha}{ }^{A} f_{F C}{ }^{\alpha}+\frac{1}{4} \eta_{A B} f_{E C}{ }^{A} f_{F D}{ }^{B}\right) \bar{\pi}^{E} \bar{\pi}^{F}+\mathcal{O}\left(\bar{\pi}^{3}\right) .
\end{aligned}
$$

For a compact group, the structure constants are completely antisymmetric, so the linear term in $\bar{\pi}$ vanishes if $\eta_{A B} \propto \delta_{A B}$. However, in some cases, such as the SM with custodial symmetry violation, the linear term is non-zero.

The geometric quantities we need can be computed directly from the metric eq. (4.28). The Christoffel symbol is

$$
\begin{aligned}
\Gamma_{B C}^{A}= & \frac{1}{2} \eta^{A G}\left(\eta_{C E} f_{B G}^{E}+\eta_{B E} f_{C G}{ }^{E}\right) \\
& +\frac{1}{4}\left(f_{G B}{ }^{E} \eta_{E C}+f_{G C}{ }^{E} \eta_{E B}\right)\left(f_{D H}{ }^{A} \eta^{H G}+f_{D H}{ }^{G} \eta^{A H}\right) \bar{\pi}^{D} \\
& -\frac{1}{4}\left(f_{H C}{ }^{G} f_{D B}{ }^{E}+f_{D C}{ }^{G} f_{H B}{ }^{E}\right) \eta^{A H} \eta_{G E} \bar{\pi}^{D} \\
& +\frac{1}{12}\left(f_{C \alpha}{ }^{A} f_{D B}{ }^{\alpha}+f_{B \alpha}{ }^{A} f_{D C}{ }^{\alpha}\right) \bar{\pi}^{D} \\
& +\frac{1}{4}\left(f_{C G}{ }^{\alpha} \eta_{B E}+f_{B G}{ }^{\alpha} \eta_{C E}\right) \eta^{A G} f_{D \alpha}{ }^{E} \bar{\pi}^{D}+\mathcal{O}\left(\bar{\pi}^{2}\right),
\end{aligned}
$$

where $\eta^{A B}$ is the inverse of $\eta_{A B}$, and the Jacobi identity has been used to simplify the final result. The Riemann curvature tensor is

$$
\begin{aligned}
\frac{1}{F_{\pi}^{2}} R_{A B C D}= & \frac{1}{4}\left(f_{A B}{ }^{\alpha} f_{D \alpha}{ }^{E} \eta_{C E}-f_{A B}{ }^{\alpha} f_{C \alpha}{ }^{E} \eta_{D E}+f_{C D}{ }^{\alpha} f_{B \alpha}{ }^{E} \eta_{A E}-f_{C D}{ }^{\alpha} f_{A \alpha}{ }^{E} \eta_{B E}\right) \\
& +\frac{1}{4}\left(f_{A D}{ }^{G} f_{B C}{ }^{E}-f_{A C}{ }^{G} f_{B D}{ }^{E}-2 f_{A B}{ }^{G} f_{C D}{ }^{E}\right) \eta_{G E} \\
& +\frac{1}{4} \eta^{G E}\left[\left(f_{A G}{ }^{H} \eta_{D H}+f_{D G}{ }^{H} \eta_{A H}\right)\left(f_{B E}{ }^{I} \eta_{C I}+f_{C E}{ }^{I} \eta_{B I}\right)\right. \\
& \left.-\left(f_{B G}{ }^{H} \eta_{D H}+f_{D G}{ }^{H} \eta_{B H}\right)\left(f_{A E}{ }^{I} \eta_{C I}+f_{C E}{ }^{I} \eta_{A I}\right)\right]
\end{aligned}
$$


where we recall that the sum on $\alpha$ runs over both broken and unbroken generators, whereas the sums on $E$, etc. are only over the broken generators. The Ricci curvature is

$$
\begin{aligned}
R_{B D}= & \frac{1}{4}\left(f_{A B}{ }^{\alpha} f_{D \alpha}{ }^{A}+f_{A D}{ }^{\alpha} f_{B \alpha}{ }^{A}-f_{A B}{ }^{\alpha} f_{C \alpha}{ }^{G} \eta_{D G} \eta^{A C}-f_{A D}{ }^{\alpha} f_{C \alpha}{ }^{G} \eta_{B G} \eta^{A C}\right) \\
& -\frac{3}{4} f_{A B}{ }^{G} f_{C D}{ }^{H} \eta^{A C} \eta_{G H} \\
& +\frac{1}{4} \eta^{G H}\left(f_{A G}{ }^{R} \eta_{D R}+f_{D G}{ }^{R} \eta_{A R}\right)\left(f_{B H}{ }^{R} \eta_{C R}+f_{C H}{ }^{R} \eta_{B R}\right) \eta^{A C} \\
& -\frac{1}{2}\left(f_{B G}{ }^{R} \eta_{D R}+f_{D G}{ }^{R} \eta_{B R}\right) f_{A H}{ }^{A} \eta^{G H},
\end{aligned}
$$

and the scalar curvature is

$$
F_{\pi}^{2} R=f_{A B}{ }^{\alpha} f_{C \alpha}{ }^{A} \eta^{B C}-\frac{1}{4} f_{A B}{ }^{C} f_{G H}{ }^{D} \eta^{A G} \eta^{B H} \eta_{C D}+\frac{1}{2} f_{A C}{ }^{B} f_{B D}{ }^{A} \eta^{C D}-f_{A C}{ }^{A} f_{B D}{ }^{B} \eta^{C D}
$$

The scalar curvature does not have a definite sign unless the group is compact. Eqs. (4.28), (4.29), (4.30), (4.31) and (4.32) are valid even for non-reductive cosets.

The results simplify considerably in a number of special cases. For a symmetric coset, $f_{A B}{ }^{C}=0$, and the curvatures eqs. (4.30), (4.31) and (4.32) reduce to

$$
\begin{aligned}
\frac{1}{F_{\pi}^{2}} R_{A B C D} & =\frac{1}{4}\left(f_{A B}{ }^{\alpha} f_{D \alpha}{ }^{G} \eta_{C G}-f_{A B}{ }^{\alpha} f_{C \alpha}{ }^{G} \eta_{D G}+f_{C D}{ }^{\alpha} f_{B \alpha}{ }^{G} \eta_{A G}-f_{C D}{ }^{\alpha} f_{A \alpha}{ }^{G} \eta_{B G}\right) \\
R_{B D} & =\frac{1}{4}\left(f_{A B}{ }^{\alpha} f_{D \alpha}{ }^{A}+f_{A D}{ }^{\alpha} f_{B \alpha}{ }^{A}-f_{A B}{ }^{\alpha} f_{C \alpha}{ }^{G} \eta_{D G} \eta^{A C}-f_{A D}{ }^{\alpha} f_{C \alpha}{ }^{G} \eta_{B G} \eta^{A C}\right) \\
F_{\pi}^{2} R & =f_{A B}{ }^{\alpha} f_{C \alpha}{ }^{A} \eta^{B C}
\end{aligned}
$$

where the sum on $\alpha=\{a, A\}$ can be restricted to the unbroken generator index $a$ only.

Another special case is $\mathcal{G}$ compact and $\eta_{A B}=\delta_{A B}$. For a compact group, the generators can be normalized so that $\operatorname{Tr} t_{\alpha} t_{\beta} \propto \delta_{\alpha \beta}$, so the structure constants are completely antisymmetric tensors in their three indices. Writing the structure constants with three lower indices in the usual notation for compact groups, eqs. (4.30), (4.31) and (4.32) simplify to

$$
\begin{aligned}
\frac{1}{F_{\pi}^{2}} R_{A B C D} & =f_{A B \alpha} f_{C D \alpha}-\frac{3}{4} f_{A B G} f_{C D G}=f_{A B g} f_{C D g}+\frac{1}{4} f_{A B G} f_{C D G}, \\
R_{B D} & =f_{A B g} f_{A D g}+\frac{1}{4} f_{A B G} f_{A D G} \\
F_{\pi}^{2} R & =f_{A B g} f_{A B g}+\frac{1}{4} f_{A B G} f_{A B G} .
\end{aligned}
$$

An interesting feature is the relative $1 / 4$ for the sum over broken generator index $G$ relative to the sum over unbroken generator index $g$. 
If one adds the additional restriction that the coset of the compact group $\mathcal{G}$ is symmetric, so $f_{A B C}=0$, the formulæ eqs. (4.34) simplify further to

$$
\begin{aligned}
\frac{1}{F_{\pi}^{2}} R_{A B C D} & =f_{A B g} f_{C D g} \\
R_{B D} & =f_{A B g} f_{A D g}=\frac{1}{2} C_{A}(\mathcal{G}) \delta_{B D}, \\
F_{\pi}^{2} R & =\frac{1}{2} C_{A}(\mathcal{G}) N_{\pi}
\end{aligned}
$$

where $C_{A}(\mathcal{G})$ is the Casimir in the adjoint representation of $\mathcal{G}$, and $N_{\pi}=\operatorname{dim} \mathcal{G} / \mathcal{H}$ is the number of broken generators.

Finally, if the gauge group is compact and completely broken, so that $\mathcal{G} / \mathcal{H}=\mathcal{G}$, and $\eta_{A B}=\delta_{A B}$, eqs. (4.34) become

$$
\begin{aligned}
\frac{1}{F_{\pi}^{2}} R_{A B C D} & =\frac{1}{4} f_{A B G} f_{C D G}, \\
R_{B D} & =\frac{1}{4} C_{A}(\mathcal{G}) \delta_{B D} \\
F_{\pi}^{2} R & =\frac{1}{4} C_{A}(\mathcal{G}) N_{\pi} .
\end{aligned}
$$

\subsection{Matter fields}

We refer to all non-Goldstone boson or gauge fields generically as matter fields. The CCWZ transformation for matter fields $\psi$ under the group transformation law eq. (4.12) is

$$
\psi \rightarrow D^{(\psi)}(h) \psi
$$

where $D^{(\psi)}(h)$ are the $\mathcal{H}$ representation matrices for $\psi$. Note that $D^{(\psi)}(h)$ is assumed to be an irreducible representation, so if it is reducible, one must first decompose it into its irreducible representations. The different irreducible representation components are then treated as separate matter fields. One can define a chiral covariant derivative for matter field $\psi$ by

$$
D_{\mu} \psi \rightarrow\left(\partial_{\mu}+i T_{a}^{(\psi)} V_{\mu}^{a}\right) \psi
$$

where $T_{a}^{(\psi)}$ are the generators of the unbroken subgroup $\mathcal{H}$ in the representation $D^{(\psi)}(h)$ of $\mathcal{H}$. The chiral covariant derivative transforms as

$$
\left(D_{\mu} \psi\right) \rightarrow D^{(\psi)}(h)\left(D_{\mu} \psi\right)
$$

The covariant derivative eq. (4.38) is derived in CCWZ. The argument relies on defining it as the ordinary derivative at $\xi=1$, and then using $\mathcal{G}$ action to define it for arbitrary $\xi$. The key point (which is not true for non-reductive cosets) is that if $g \in H$, then $h$ in eq. (4.12) is a constant, so the ordinary derivative transforms the same way as the field, eq. (4.37). Using this result at $\xi=1$, the transformation eq. (4.39) for arbitrary $\xi$ follows.

The covariant derivative eq. (4.38) is based on eq. (4.16), and hence on the MaurerCartan form $g^{-1} \mathrm{~d} g$. This is the canonical connection on the principal $\mathcal{H}$-bundle $\mathcal{G} \rightarrow \mathcal{G} / \mathcal{H}$, 
and makes no reference to a metric, i.e. to $\eta_{A B}$. One can also define covariant derivatives based on the metric (Christoffel) connection eq. (4.29), which does depend on $\eta_{A B}$. The two are equivalent if $\eta_{A B}=\delta_{A B}$, i.e. if the $\mathcal{G}$-invariant metric on $\mathcal{G} / \mathcal{H}$ is obtained from a $\mathcal{G}$-invariant metric on $\mathcal{G}$. The difference in the connections transforms as a $\mathcal{H}$-invariant tensor [62], so that the change in connection can be compensated by a change in coefficients of invariant terms in the sigma model Lagrangian. The exponential map $\xi(\lambda)=\exp (X \lambda)$ is geodesic for the Maurer-Cartan connection, but not for a general $\eta_{A B}$ metric connection.

\subsection{Sectional curvature}

The sectional curvature $K(Y, Z)$ in the plane spanned by tangent vectors $Y$ and $Z$ is defined by

$$
K(Y, Z)=\frac{R_{A B C D} Y^{A} Z^{B} Y^{C} Z^{D}}{\langle Y, Y\rangle\langle Z, Z\rangle-\langle Y, Z\rangle^{2}}
$$

where the inner product $\langle *, *\rangle$ is w.r.t. the metric $g_{A B}$. The Cauchy-Schwartz inequality implies the denominator is positive, so the sign of the sectional curvature depends on the sign of the numerator. The sign of the sectional curvature is important, because, as shown in refs. [1, 33, 60], the sign of deviations in Higgs-gauge boson scattering amplitudes from SM amplitudes is determined by the sign of the sectional curvatures of the HEFT sigma model.

From eq. (4.30),

$$
\begin{aligned}
\frac{1}{F_{\pi}^{2}} R_{A B C D} Y^{A} Z^{B} Y^{C} Z^{D}=\frac{1}{2} f_{Y Z}{ }^{\alpha}\left(f_{Z \alpha}{ }^{A} Y^{B} \eta_{A B}-f_{Y \alpha}{ }^{A} Z^{B} \eta_{A B}\right)-\frac{3}{4} f_{Y Z}{ }^{A} f_{Y Z}{ }^{B} \eta_{A B} \\
+\frac{1}{4}\left(f_{Y G}{ }^{A} Z^{B} \eta_{A B}+f_{Z G}{ }^{A} Y^{B} \eta_{A B}\right)\left(f_{Y H}{ }^{C} Z^{D} \eta_{C D}+f_{Z H}{ }^{C} Y^{D} \eta_{C D}\right) \eta^{G H} \\
-f_{Y G}{ }^{A} f_{Z H}{ }^{C} Y^{B} Z^{D} \eta_{A B} \eta_{C D} \eta^{G H}
\end{aligned}
$$

and we have used the definition

$$
[Y, Z]=\left[Y^{A} T_{A}, Z^{B} T_{B}\right] \equiv f_{Y Z}{ }^{\alpha} t_{\alpha}
$$

for $f_{Y Z}{ }^{\alpha}$. The general form eq. (4.41) does not have a definite sign.

For compact groups with $\eta_{A B} \propto \delta_{A B}$, antisymmetry of the structure constants implies

$$
\frac{1}{F_{\pi}^{2}} R_{A B C D} Y^{A} Z^{B} Y^{C} Z^{D}=f_{Y Z}^{g} f_{Y Z}^{g}+\frac{1}{4} f_{Y Z}{ }^{G} f_{Y Z}{ }^{G} \geq 0
$$

is positive definite for any pair of vectors $Y, Z$. For compact groups with $\eta_{A B} \neq \delta_{A B}$, the sectional curvatures need not be positive. A simple example is $\mathcal{G}=\mathrm{SU}(2)$ completely broken, with $\eta_{A B}=\operatorname{diag}\left(\eta_{1}, \eta_{2}, \eta_{3}\right)$, and $Y=(1,0,0), Z=(0,1,0)$, in which case

$$
K(Y, Z)=\frac{2\left(\eta_{1}+\eta_{2}\right) \eta_{3}+\left(\eta_{1}-\eta_{2}\right)^{2}-3 \eta_{3}^{2}}{4 F_{\pi}^{2} \eta_{1} \eta_{2} \eta_{3}}
$$

which is negative for $\eta_{3} \gg \eta_{1,2}$.

In HEFT applications where there is only a single $h$ field, the possible sectional curvatures are: 
(a) Both $Y$ and $Z$ are in the Goldstone boson directions. Since the Goldstone boson manifold $S^{3}$ is a maximally symmetric space, $K\left(Y_{\pi}, Z_{\pi}\right)$ is independent of the choice $Y_{\pi}, Z_{\pi}$, and is the quantity $K\left(Y_{\pi}, Z_{\pi}\right)=\mathfrak{R}_{4}$ in ref. [1].

(b) $Y$ is in the Goldstone boson direction, and $Z$ is in the $h$ direction. In this case $K\left(Y_{\pi}, Z_{h}\right)$ is independent of the choice $Y_{\pi}$ and $Z_{h}$ (since there is only one direction $\left.Z_{h}\right)$ and is $K\left(Y_{\pi}, Z_{h}\right)=\mathfrak{R}_{2 h}$ in ref. [1].

As shown in ref. [1], deviations in $W_{L} W_{L} \rightarrow W_{L} W_{L}$ were proportional to $\mathfrak{r}_{4}=\mathfrak{R}_{4}(h=$ 0 ), the sectional curvature where $Y$ and $Z$ are in Goldstone boson directions. The longitudinal gauge bosons at high energies are related to the Goldstone bosons, and so probe the Goldstone boson directions in $\mathcal{M}$. The $W_{L} W_{L} \rightarrow h h$ scattering amplitudes is proportional to $\mathfrak{r}_{2 h}=\mathfrak{R}_{2 h}(h=0)$, and probes the sectional curvature where $Y$ is in a Goldstone boson direction, and $Z$ in the Higgs direction. If the HEFT is based on a composite Higgs theory [28], where $h$ is itself a (pseudo) Goldstone boson of some strong dynamics at a scale $f>v$, then we see from eq. (4.43) that $\mathfrak{R}_{4}$ and $\mathfrak{R}_{2 h}$ are both positive if the composite Higgs model is based on a compact group. On the other hand, if the sigma-model group is non-compact, it is possible to get negative values [33] for these curvatures because eq. (4.41) has no definite sign.

We also consider multi-Higgs theories in section 6 . In such theories, the possible sectional curvatures are $\mathfrak{R}_{4}=K\left(Y_{\pi}, Z_{\pi}\right), \mathfrak{R}_{2 h, I}=K\left(Y_{\pi}, Z_{I}\right)$, where $Z_{I}$ runs over the possible Higgs directions, and $K\left(Y_{I}, Z_{J}\right)$ over distinct pairs of Higgs directions $I \neq J$.

\section{The Standard Model and custodial symmetry violation}

The SM sigma model for the custodial symmetric breaking pattern $\mathrm{SU}(2)_{L} \times \mathrm{SU}(2)_{R} \rightarrow$ $\mathrm{SU}(2)_{V}$ can be written in the CCWZ formalism, choosing the broken generators to be $T_{L}$. Let

$$
\mathrm{U}(x)=e^{i \bar{\pi}^{A}(x) T_{A}}
$$

be a $2 \times 2$ matrix, where $T_{A}$ are $\mathrm{SU}(2)_{L}$ generators, and $\bar{\pi}^{A}$ are dimensionless.

The $\xi$ field of the CCWZ formalism given by exponentiating the broken generators is

$$
\xi(x)=\left(\begin{array}{cc}
\mathrm{U}(x) \\
0 & 1_{2 \times 2}
\end{array}\right),
$$

where the first $2 \times 2$ block is the $\mathrm{SU}(2)_{L}$ transformation, and the second is the $\mathrm{SU}(2)_{R}$ 
transformation. From this $\xi$ field, one finds

$$
\begin{aligned}
\xi(x)^{-1} D_{\mu} \xi & =\left(\begin{array}{cc}
\mathrm{U}(x)^{-1} & \\
0 & 1_{2 \times 2}
\end{array}\right)\left[\left(\begin{array}{cc}
\partial_{\mu} \mathrm{U}(x) \\
0 & 0
\end{array}\right)+\left(\begin{array}{cc}
i g_{2} W_{\mu}^{\alpha} T_{\alpha} \mathrm{U}(x) & \\
0 & i g_{1} B_{\mu} T_{3}
\end{array}\right)\right] \\
& =\left(\begin{array}{cc}
\mathrm{U}(x)^{-1} \partial_{\mu} \mathrm{U}(x)+\mathrm{U}(x)^{-1} i g_{2} W_{\mu}^{\alpha} T_{\alpha} \mathrm{U}(x) & \\
0 & i g_{1} B_{\mu} T_{3}
\end{array}\right) \\
= & \left(\begin{array}{cc}
i g_{1} B_{\mu} T_{3} & \\
0 & i g_{1} B_{\mu} T_{3}
\end{array}\right) \\
& +\left(\begin{array}{cc}
\mathrm{U}(x)^{-1} \partial_{\mu} \mathrm{U}(x)+\mathrm{U}(x)^{-1} i g_{2} W_{\mu}^{\alpha} T_{\alpha} \mathrm{U}(x)-i g_{1} B_{\mu} T_{3} \\
0
\end{array}\right),
\end{aligned}
$$

where the last line projects onto the unbroken and broken spaces, respectively. Thus, we obtain

$$
i\left(D_{\mu} \bar{\pi}\right)^{A} T_{A}=\mathrm{U}(x)^{-1} \partial_{\mu} \mathrm{U}(x)+\mathrm{U}(x)^{-1} i g_{2} W_{\mu}^{\alpha} T_{\alpha} \mathrm{U}(x)-i g_{1} B_{\mu} T_{3},
$$

and, using the results in appendix A,

$$
\begin{aligned}
\left(D_{\mu} \bar{\pi}\right)^{A}= & \left(\frac{\sin |\bar{\pi}|}{|\bar{\pi}|}\right) \mathrm{d} \bar{\pi}^{A}+\left(\frac{1-\cos |\bar{\pi}|}{|\bar{\pi}|^{2}}\right) \epsilon_{A B C} \bar{\pi}^{B} \mathrm{~d} \bar{\pi}^{C}+\left(\frac{|\bar{\pi}|-\sin |\bar{\pi}|}{|\bar{\pi}|^{3}}\right) \bar{\pi}^{A}(\overline{\boldsymbol{\pi}} \cdot \mathbf{d} \overline{\boldsymbol{\pi}}) \\
& +g_{2} W_{\mu}^{A} \cos |\bar{\pi}|+g_{2}\left(\frac{\sin |\bar{\pi}|}{|\bar{\pi}|}\right) \epsilon_{A B C} \bar{\pi}^{B} W_{\mu}^{C} \\
& +g_{2}\left(\frac{1-\cos |\bar{\pi}|}{|\bar{\pi}|^{2}}\right)\left(\overline{\boldsymbol{\pi}} \cdot \boldsymbol{W}_{\mu}\right) \bar{\pi}^{A}-g_{1} B_{\mu} \delta_{A 3}
\end{aligned}
$$

with $|\bar{\pi}|^{2}=\bar{\pi} \cdot \bar{\pi}$. Decomposing $\left(D_{\mu} \bar{\pi}\right)^{A}$ into gauge and non-gauge pieces as in eq. (4.19) yields

$$
\left(D_{\mu} \bar{\pi}\right)^{A}=e^{A}{ }_{B} \partial_{\mu} \bar{\pi}^{B}+F_{\beta}^{A} W_{\mu}^{\beta}+F_{Z}^{A} Z_{\mu}+F_{\gamma}^{A} A_{\mu},
$$

where

$$
\begin{aligned}
e_{B}^{A} & =\left(\frac{\sin |\bar{\pi}|}{|\bar{\pi}|}\right) \delta_{B}^{A}-\left(\frac{1-\cos |\bar{\pi}|}{|\bar{\pi}|^{2}}\right) \epsilon_{A B C} \bar{\pi}^{C}+\left(\frac{|\bar{\pi}|-\sin |\bar{\pi}|}{|\bar{\pi}|^{3}}\right) \bar{\pi}^{A} \bar{\pi}^{B}, \\
F_{\beta}^{A} & =\frac{e}{s_{W}}\left[\delta_{\beta}^{A} \cos |\bar{\pi}|+\left(\frac{\sin |\bar{\pi}|}{|\bar{\pi}|}\right) \epsilon_{A D \beta} \bar{\pi}^{D}+\left(\frac{1-\cos |\bar{\pi}|}{|\bar{\pi}|^{2}}\right) \bar{\pi}^{\beta} \bar{\pi}^{A}\right], \quad \beta=1,2 \\
F_{Z}^{A} & =\frac{e}{s_{W} c_{W}}\left[\delta_{3}^{A}\left(s_{W}^{2}+c_{W}^{2} \cos |\bar{\pi}|\right)+c_{W}^{2}\left(\frac{\sin |\bar{\pi}|}{|\bar{\pi}|}\right) \epsilon_{A B 3} \bar{\pi}^{B}+c_{W}^{2}\left(\frac{1-\cos |\bar{\pi}|}{|\bar{\pi}|^{2}}\right) \bar{\pi}^{3} \bar{\pi}^{A}\right], \\
F_{\gamma}^{A} & =e\left[-\delta_{3}^{A}(1-\cos |\bar{\pi}|)+\left(\frac{\sin |\bar{\pi}|}{|\bar{\pi}|}\right) \epsilon_{A B 3} \bar{\pi}^{B}+\left(\frac{1-\cos |\bar{\pi}|}{|\bar{\pi}|^{2}}\right) \bar{\pi}^{3} \bar{\pi}^{A}\right],
\end{aligned}
$$


with $c_{W}=\cos \theta_{W}$ and $s_{W}=\sin \theta_{W}$. The $F_{\alpha}^{A}$ can be used to construct the Killing vectors using eq. (4.24). Expanding these equations gives

$$
\begin{aligned}
e^{A}{ }_{B} & =\delta_{B}^{A}-\frac{1}{2} \epsilon_{A B C} \bar{\pi}^{C}+\frac{1}{6}\left[\bar{\pi}^{A} \bar{\pi}^{B}-|\bar{\pi}|^{2} \delta_{B}^{A}\right]+\ldots \\
F_{\beta}^{A} & =\frac{e}{s_{W}}\left[\delta_{\beta}^{A}\left(1-\frac{1}{2}|\bar{\pi}|^{2}\right)+\epsilon_{A D \beta} \bar{\pi}^{D}+\frac{1}{2} \bar{\pi}^{\beta} \bar{\pi}^{A}\right]+\ldots, \quad \beta=1,2 \\
F_{Z}^{A} & =\frac{e}{s_{W} c_{W}}\left[\delta_{3}^{A}\left(1-\frac{1}{2} c_{W}^{2}|\bar{\pi}|^{2}\right)+c_{W}^{2} \epsilon_{A B 3} \bar{\pi}^{B}+\frac{1}{2} c_{W}^{2} \bar{\pi}^{3} \bar{\pi}^{A}\right]+\ldots, \\
F_{\gamma}^{A} & =e\left[-\frac{1}{2}|\bar{\pi}|^{2} \delta_{3}^{A}+\epsilon_{A B 3} \bar{\pi}^{B}+\frac{1}{2} \bar{\pi}^{3} \bar{\pi}^{A}\right]+\ldots
\end{aligned}
$$

In unitary gauge, $\bar{\pi}=0$ and

$$
F_{\beta}^{A}=\frac{e}{s_{W}} \delta_{\beta}^{A}, \quad \beta=1,2, \quad F_{Z}^{A}=\frac{e}{s_{W} c_{W}} \delta_{3}^{A}, \quad F_{\gamma}^{A}=0,
$$

so the photon is massless, and $W, Z$ acquire mass.

The most general $\mathcal{O}\left(p^{2}\right)$ Lagrangian is

$$
L=\frac{1}{2} \sum_{A B} \eta_{A B}\left(D_{\mu} \bar{\pi}\right)^{A}\left(D_{\mu} \bar{\pi}\right)^{B}
$$

where $\eta_{A B}$ is a $\mathcal{H}$-invariant tensor. For the $\mathrm{SM}$ with custodial $\mathrm{SU}(2)$ symmetry, the breaking pattern is $\mathrm{SU}(2)_{L} \times \mathrm{SU}(2)_{R} \rightarrow \mathrm{SU}(2)_{V}$. The tensor $\eta_{A B}$ must be invariant under the unbroken $\mathcal{H}=\mathrm{SU}(2)_{V}$ symmetry, so

$$
\eta_{A B}=\frac{v^{2}}{8} \delta_{A B}
$$

where $v \sim 246 \mathrm{GeV}$ is chosen to give the correct gauge boson masses.

If custodial symmetry is not exact, the breaking pattern is $\mathrm{SU}(2)_{L} \times \mathrm{U}(1)_{Y} \rightarrow \mathrm{U}(1)_{\mathrm{em}}$, and $\eta_{A B}$ must be invariant under the unbroken $\mathcal{H}=\mathrm{U}(1)_{\text {em }}$ symmetry. In this case,

$$
\eta_{A B}=\frac{v^{2}}{8}\left(\begin{array}{lll}
1 & 0 & 0 \\
0 & 1 & 0 \\
0 & 0 & \rho
\end{array}\right),
$$

where $\rho$ is the $\rho$-parameter

$$
\rho=\frac{M_{Z}^{2} c_{W}^{2}}{M_{W}^{2}}
$$

which is no longer equal to one. The experimental constraint on the $\rho$ parameter is an extremely stringent constraint on custodial symmetry violation, since it requires $|\rho-1| \lesssim$ 0.01. A simple example of custodial symmetry violation is the SM with an additional triplet scalar field [63]

$$
\chi=\left[\begin{array}{cc}
\frac{1}{\sqrt{2}} \chi^{+} & -\chi^{++} \\
\chi^{0} & -\frac{1}{\sqrt{2}} \chi^{+}
\end{array}\right]
$$


If the doublet and triplet vacuum expectation values are

$$
\langle H\rangle=\left[\begin{array}{c}
0 \\
\frac{v_{D}}{\sqrt{2}}
\end{array}\right], \quad\langle\chi\rangle=\left[\begin{array}{cc}
0 & 0 \\
\frac{v_{T}}{\sqrt{2}} & 0
\end{array}\right],
$$

then the values of the $\eta_{A B}$ parameters in eq. (5.12) are

$$
v^{2}=v_{D}^{2}+2 v_{T}^{2}, \quad \rho=\frac{v_{D}^{2}+4 v_{T}^{2}}{v_{D}^{2}+2 v_{T}^{2}} .
$$

The geometry of the scalar manifold with metric eq. (5.10) has been studied in other contexts [64]. The configuration space of a rigid body with one point fixed is given by the rotation matrix $R(\theta, \phi, \psi) \in \mathrm{SO}(3)$ parameterized by three Euler angles, and, up to $\mathbb{Z}_{2}$ factors, is the same as the Goldstone boson manifold of the SM. Rotations of the body about space-fixed axes correspond to $\mathrm{SO}(3)_{L}$ rotations $R \rightarrow g_{L} R, g_{L} \in \mathrm{SO}(3)$, and rotations about the body-fixed axes correspond to $\mathrm{SO}(3)_{R}$ rotations $R \rightarrow R g_{R}^{-1}, g_{R} \in \mathrm{SO}(3)$. The body-axis angular momenta are given by $\omega^{A} T_{A}=R^{-1} \dot{R}$. The kinetic energy for a rigid body is then given by the analog of eq. (5.10),

$$
L=\frac{1}{2} \sum_{A} I_{A}\left(\omega^{A}\right)^{2}
$$

where $\eta_{A B}$ can be chosen to be diagonal by picking the body axes to coincide with the principal axes of the body. The kinetic energy for a spherical top with all three principal moments of inertia equal, $I_{1}=I_{2}=I_{3}$, is the analog of the SM with custodial symmetry. The configuration space of the top is the (undeformed) three-sphere $S^{3}$. The custodial symmetry violating case is analogous to $I_{1}=I_{2} \neq I_{3}$, which is the configuration space of a symmetric top. This space is known as the squashed three-sphere, and also occurs in the metric for the Taub universe [64]. The asymmetric top with all $I_{i}$ different would correspond to the SM with electromagnetism broken.

\section{HEFT with multiple singlet scalar bosons}

The HEFT formalism can be extended to the case of multiple singlet (under custodial $\mathrm{SU}(2))$ Higgs fields $h^{I}, I=1,2, \cdots$, which involves adding additional singlet scalars to the SM field content. The generalization of the HEFT Lagrangian eq. (2.24) to multiple singlet scalar fields is

$$
L=\frac{1}{2} v^{2} F(h)^{2}\left(\partial_{\mu} \boldsymbol{n}\right)^{2}+\frac{1}{2} g_{I J}(h)\left(\partial_{\mu} h^{I}\right)\left(\partial_{\mu} h^{J}\right)-V(h)+\ldots
$$

where $F(h)$ is an arbitrary function of the dimensionless singlet scalar fields $h^{I} / v$. The coordinates $\left\{h^{I}\right\}$ are chosen so that $h=(0,0, \ldots, 0)$ is the ground state, and the HEFT function $F(h)$ is normalized so that

$$
F(0, \ldots, 0)=1
$$

since the radius of $S^{3}$ in the vacuum is fixed to be $v$ by the gauge boson masses. 
Consider the $O(4) \rightarrow O(3)$ symmetry breaking pattern of the SM, with multiple scalar fields $h^{I}$ which are singlets under the unbroken custodial $O(3)$ symmetry. The most general metric of the scalar fields $\Phi^{i} \equiv\left\{\pi^{A}, h^{I}\right\}$ has the form

$$
g_{i j}(\Phi)=\left[\begin{array}{cc}
F(h)^{2} g_{A B}(\pi) & 0 \\
0 & g_{I J}(h)
\end{array}\right],
$$

where $\pi^{A} / v$ are coordinates on the coset space $\mathcal{G} / \mathcal{H}=O(4) / O(3)=S^{3}$, and $g_{A B}(\pi)$ is the metric on the unit 3 -sphere. $O(4)$ invariance implies that the off-diagonal metric terms $g_{A I}$ and $g_{I A}$ vanish, and that $g_{I J}(h)$ has no dependence on the $\pi$ fields. An easy way to prove that the general metric takes the form eq. (6.3) is to note that a point on $S^{3}$ is given by a four-component unit vector $\boldsymbol{n}$. The entry $g_{I J}(h)$ can depend on $\boldsymbol{n}$, but not on its derivatives; $O(4)$ invariance then requires it to be function of $\boldsymbol{n} \cdot \boldsymbol{n}=1$, and therefore independent of $\boldsymbol{\pi}$. Similarly, $g_{I A} \partial_{\mu} \pi^{A}$ is an $O(4)$ invariant function of $\boldsymbol{n}$ and $\partial_{\mu} \boldsymbol{n}$ with one derivative; the only invariant object is $\partial_{\mu} \boldsymbol{n} \cdot \boldsymbol{n}=0$, so the off-diagonal entries vanish. The 11 entry has the form $F(h)^{2} g_{A B}(\pi)$ because $\mathcal{G}$-invariance requires that $h$ dependence is an overall multiplicative factor, since there is only one $\mathcal{G}$-invariant metric on $S^{3}$. We will consider the geometry of the metric eq. (6.3), with a general metric $g_{A B}(\pi)$, so the results are valid for a general $\mathcal{G} / \mathcal{H}$ manifold as long as the off-diagonal terms of $g_{i j}(\Phi)$ vanish as in eq. (6.3).

Using the metric eq. (6.3), the Christoffel symbols are

$$
\begin{array}{lll}
\Gamma_{B C}^{A}=\gamma_{B C}^{A}, & \Gamma_{B K}^{A}=\frac{F_{, K}}{F} \delta_{B}^{A}, & \Gamma_{J K}^{A}=0, \\
\Gamma_{B C}^{I}=-F F_{, M} g^{I M} g_{B C}, & \Gamma_{B K}^{I}=0, & \Gamma_{J K}^{I}=\gamma_{J K}^{I},
\end{array}
$$

where $\gamma_{B C}^{A}$ and $\gamma_{J K}^{I}$ are the Christoffel symbols computed from the metrics $g_{A B}(\pi)$ and $g_{I J}(h)$, respectively. Similarly, in the expressions below, $r^{A}{ }_{B C D}, r_{B D}$ and $r_{\pi}$ are the curvatures computed from the metric $g_{A B}(\pi)$, whereas $r_{J K L}^{I}, r_{J L}$ and $r_{h}$ are the curvatures computed from the metric $g_{I J}(h)$. The Riemann curvature tensor is

$$
\begin{aligned}
R_{B C D}^{A} & =r_{B C D}^{A}-g^{M N} F_{, M} F_{, N}\left(\delta_{C}^{A} g_{D B}-\delta^{A}{ }_{D} g_{B C}\right), & R^{A}{ }_{B C L} & =0, \\
R_{B K L}^{A} & =0, & R_{J C D}^{I} & =0, \\
R_{J K D}^{I} & =0, & R^{I}{ }_{J K L} & =r^{I}{ }_{J K L}, \\
R_{J C D}^{A} & =0, & R^{A}{ }_{J K L} & =0, \\
R_{B C D}^{I} & =0, & R^{I}{ }_{B K D} & =-g_{D B} g^{I M} F_{; M K}, \\
R_{B K L}^{I} & =0, & R_{J C L}^{A} & =-\delta_{C}^{A} F_{j L} .
\end{aligned}
$$

The covariant derivatives of $F$ are w.r.t. $\gamma_{J K}^{I}$. The Ricci tensor is

$$
\begin{aligned}
R_{B D} & =r_{B D}-g^{R S} F_{, R} F_{, S}\left(N_{\pi}-1\right) g_{B D}-g_{B D} g^{R S} F F_{; R S}, \\
R_{B L} & =0 \\
R_{J L} & =-N_{\pi} F_{; J L}+r_{J L},
\end{aligned}
$$


and the curvature scalar is

$$
R=\frac{1}{F^{2}} r_{\pi}-N_{\pi}\left(N_{\pi}-1\right) \frac{1}{F^{2}} g^{R S} F_{, R} F_{, S}-2 N_{\pi} \frac{1}{F} g^{R S} F_{; R S}+r_{h} .
$$

If $\mathcal{G} / \mathcal{H}$ is a maximally symmetric space,

$$
r_{B C D}^{A}=\frac{1}{F_{\pi}^{2}}\left(\delta^{A}{ }_{C} g_{B D}-\delta^{A}{ }_{D} g_{B C}\right), \quad r_{B D}=\frac{1}{F_{\pi}^{2}}\left(N_{\pi}-1\right) g_{B D}, \quad r_{\pi}=\frac{1}{F_{\pi}^{2}} N_{\pi}\left(N_{\pi}-1\right) .
$$

The above expressions reduce to the formulæ given in ref. [1] for one Higgs singlet field $h$ and $\mathcal{G} / \mathcal{H}$ a symmetric space, which used

$$
g_{I J}(h)=1, \quad F(h)=1+c_{1}\left(\frac{h}{v}\right)+\frac{1}{2} c_{2}\left(\frac{h}{v}\right)^{2}+\ldots
$$

with $F_{\pi}=v$.

The above expressions can be further simplified if one picks one $h$ field to be the radius of $S^{3}, F(\{h\})=h^{1}$, in which case $F$ does not depend on $h^{I}, I \neq 1$. The radial direction is in general not a mass-eigenstate direction. Letting $\rho$ be the radial direction, with $\rho=1$ in the vacuum, and letting the remaining directions still be called $h^{I}$ (there is one less $h$ now), with $I, J, K$ running over $\rho,\{h\}$, one gets a simpler version of the above equations, where $F_{, K}=1$ if $K=\rho$, and zero otherwise. For example,

$$
F \rightarrow \rho, \quad G^{R S} F_{, R} F_{, S} \rightarrow G^{\rho \rho}, \quad F_{; R S} \rightarrow-\gamma_{R S}^{\rho},
$$

etc.

\section{Conclusions}

In this paper, we have discussed the relation between the SM and two of its generalizations, SMEFT and HEFT, and have shown that HEFT can be written in SMEFT form if and only if there is an $O(4)$ invariant fixed point of the scalar manifold in a neighborhood of which the scalar fields transform as a vector of $O(4)$. We have shown that the SM can be written using scalar fields transforming either linearly or non-linearly under $\mathrm{SU}(2)_{L} \times \mathrm{U}(1)_{Y}$, and is renormalizable with either choice. Whether "the Higgs transforms linearly or non-linearly" is not observable; the correct question, which can be resolved experimentally, is whether the SM scalar manifold $\mathcal{M}$ is flat or curved.

We have discussed the formulation of scalar fields on a curved manifold, including the case with gauge symmetry, reviewed the computation of one-loop corrections in terms of the curvature, and applied these known results to the case where the manifold is a coset. The general expressions were used to obtain the one-loop renormalization of HEFT [1, 16], and details of the computation are given here.

Deviations of Higgs and longitudinal gauge boson scattering amplitudes from their SM values are given by sectional curvatures of the scalar manifold. In simple examples based on $\mathcal{G} / \mathcal{H}$ symmetry breaking with compact groups, the sectional curvatures are positive, which fixes the signs of deviations from the SM. We are investigating examples where sectional curvatures can be negative, and have given the generalization of the CCWZ formalism to non-compact groups. 


\section{Acknowledgments}

AM would like to thank Luis Alvarez-Gaumé, Luca Merlo, and Vyacheslav Rychkov for helpful discussions. This work was partially supported by grants from the Simons Foundation (\#340282 to Elizabeth Jenkins and \#340281 to Aneesh Manohar) and by DOE grant DE-SC0009919. RA would like to thank the CERN theory group for hospitality and funding.

\section{A Exponential parametrization of the $O(N)$ model}

The real antisymmetric Goldstone boson matrix is given by

$$
\boldsymbol{\Pi} \equiv i(\overline{\boldsymbol{\pi}} \cdot \boldsymbol{X})=\left[\begin{array}{cc}
0 & \overline{\boldsymbol{\pi}} \\
-\overline{\boldsymbol{\pi}}^{T} & 0
\end{array}\right]=\left[\begin{array}{cccc}
0 & \ldots & 0 & \bar{\pi}^{1} \\
0 & \ldots & 0 & \bar{\pi}^{2} \\
\vdots & & \vdots & \vdots \\
0 & \ldots & 0 & \bar{\pi}^{N_{\varphi}} \\
-\bar{\pi}^{1} & \ldots & -\bar{\pi}^{N_{\varphi}} & 0
\end{array}\right]
$$

where $\bar{\pi}^{A} \equiv \pi^{A} / F_{\pi} . \xi$ is

$$
\xi \equiv e^{\boldsymbol{\Pi}}=\mathbf{1}+\left(\frac{\sin |\bar{\pi}|}{|\bar{\pi}|}\right) \boldsymbol{\Pi}+\left(\frac{1-\cos |\bar{\pi}|}{|\bar{\pi}|^{2}}\right) \boldsymbol{\Pi}^{2}, \quad|\bar{\pi}|^{2} \equiv \bar{\pi}^{A} \bar{\pi}^{A} .
$$

The Maurer-Cartan form is

$$
\begin{aligned}
\xi^{-1} \partial_{\mu} \xi & =\left(\frac{\sin |\bar{\pi}|}{|\bar{\pi}|}\right) i\left(\partial_{\mu} \overline{\boldsymbol{\pi}}\right) \cdot \boldsymbol{X}+\left(\frac{|\bar{\pi}|-\sin |\bar{\pi}|}{|\bar{\pi}|^{3}}\right)\left(\bar{\pi}^{B} \partial_{\mu} \bar{\pi}^{B}\right) i \overline{\boldsymbol{\pi}} \cdot \boldsymbol{X} \\
& +\left(\frac{1-\cos |\bar{\pi}|}{|\bar{\pi}|^{2}}\right)\left[\begin{array}{cc}
-\left(\partial_{\mu} \bar{\pi}^{A}\right) \bar{\pi}^{B}+\bar{\pi}^{A}\left(\partial_{\mu} \bar{\pi}^{B}\right) & 0 \\
0 & 0
\end{array}\right],
\end{aligned}
$$

where the first two terms are linear combinations of the broken generators, and the last term is a linear combination of the unbroken generators. The indices $A, B$ in the last term are the row and column indices of the $N_{\varphi} \times N_{\varphi}$ submatrix in the upper 11 block. Using eq. (4.16),

$$
\left(D_{\mu} \bar{\pi}\right)^{A}=\left(\frac{\sin \bar{\pi}}{\bar{\pi}}\right)\left(\partial_{\mu} \bar{\pi}\right)^{A}+\left(\frac{\bar{\pi}-\sin \bar{\pi}}{\bar{\pi}^{3}}\right)\left(\bar{\pi}^{B} \partial_{\mu} \bar{\pi}^{B}\right) \bar{\pi}^{A}
$$

and

$$
\left.\xi^{-1} \partial_{\mu} \xi\right|_{T}=i \boldsymbol{V}_{\mu} \cdot \boldsymbol{T}=\left(\frac{1-\cos \bar{\pi}}{\bar{\pi}^{2}}\right)\left[\begin{array}{cc}
-\left(\partial_{\mu} \bar{\pi}^{A}\right) \bar{\pi}^{B}+\bar{\pi}^{A}\left(\partial_{\mu} \bar{\pi}^{B}\right) & 0 \\
0 & 0
\end{array}\right]
$$

\section{B One-loop renormalization of HEFT}

In this appendix, we provide some intermediate results in the computation of the one-loop renormalization of HEFT $[1,16]$. 
The metric for the scalar manifold $\mathcal{M}$ in HEFT is

$$
g_{i j}(\phi)=\left[\begin{array}{cc}
v^{2} F(h)^{2} g_{a b}(\bar{\pi}) & 0 \\
0 & 1
\end{array}\right],
$$

where $F(h)$ is a dimensionless function with a power series expansion in $h / v$, and $g_{a b}(\bar{\pi})$ is the metric on the Goldstone boson manifold $\mathcal{G} / \mathcal{H}=S^{3}$. The field $h$ has mass dimension one, $\bar{\pi}$ is dimensionless, and $i$ runs over indices $a, h$. The scalar kinetic term in HEFT is given by

$$
\begin{aligned}
L & =\frac{1}{2} g_{i j}(\phi) \partial_{\mu} \phi^{i} \partial^{\mu} \phi^{j}=\frac{1}{2} v^{2} F(h)^{2} g_{a b}(\bar{\pi}) \partial_{\mu} \bar{\pi}^{a} \partial^{\mu} \bar{\pi}^{b}+\frac{1}{2} \partial_{\mu} h \partial^{\mu} h \\
& \equiv \frac{1}{2} F(h)^{2} v^{2} \partial_{\mu} \boldsymbol{n} \cdot \partial^{\mu} \boldsymbol{n}+\frac{1}{2} \partial_{\mu} h \partial^{\mu} h,
\end{aligned}
$$

where the unit vector $\boldsymbol{n}(\bar{\pi})$ is a dimensionless function of the the three independent coordinates $\bar{\pi}^{a}=\pi^{a} / v$ on $S^{3}$. Note that we have chosen to normalize $\bar{\pi}^{a}$ to be dimensionless coordinates, which differs from ref. [1] by a rescaling by $v$. Eq. (B.2) implies that the $S^{3}$ metric $g_{a b}(\bar{\pi})$ is given in terms of the unit vector $\boldsymbol{n}(\bar{\pi})$ by

$$
g_{a b}(\bar{\pi}) \equiv \frac{\partial \boldsymbol{n}(\bar{\pi})}{\partial \bar{\pi}^{a}} \cdot \frac{\partial \boldsymbol{n}(\bar{\pi})}{\partial \bar{\pi}^{b}} .
$$

The Riemann curvature tensor $R_{i j k l}(\phi)$ obtained from the scalar metric $g_{i j}(\phi)$ consists of the non-vanishing components

$$
\begin{aligned}
& R_{a b c d}(\phi)=\left[1-v^{2}\left(F^{\prime}(h)\right)^{2}\right] v^{2} F(h)^{2}\left(g_{a c}(\bar{\pi}) g_{b d}(\bar{\pi})-g_{a d}(\bar{\pi}) g_{b c}(\bar{\pi})\right), \\
& R_{a h b h}(\phi)=-v^{2} F(h) F^{\prime \prime}(h) g_{a b}(\bar{\pi}),
\end{aligned}
$$

and components related to these by the permutation symmetry of the Riemann tensor. $R_{a b c d}(\phi)$ is proportional to the tensor $\left(g_{a c} g_{b d}-g_{a d} g_{b c}\right)$ because $S^{3}$ is a maximally symmetric space.

The quantities $X$ and $Y_{\mu \nu}$ from eqs. (3.44) and (3.45) that appear in the one-loop correction eq. (3.43) contain terms depending on the Riemann curvature tensor. The Riemann curvature tensor components contributing to $[X]^{i}{ }_{k}$ and $\left[Y_{\mu \nu}\right]_{j}^{i}$, respectively, are

$$
\begin{aligned}
& R_{j k l}^{i}\left(D_{\mu} \phi\right)^{j}\left(D^{\mu} \phi\right)^{l} \\
& =\left[\begin{array}{cc}
{\left[1-v^{2}\left(F^{\prime}\right)^{2}\right]\left[\left(D_{\mu} \bar{\pi}\right)^{2} \delta_{c}^{a}-\left(D_{\mu} \bar{\pi}\right)^{a}\left(D_{\mu} \bar{\pi}\right)_{c}\right]-\frac{F^{\prime \prime}}{F}\left(\partial_{\mu} h\right)\left(\partial^{\mu} h\right) \delta_{c}^{a}} & \frac{F^{\prime \prime}}{F}\left(D_{\mu} \bar{\pi}\right)^{a}\left(\partial_{\mu} h\right) \\
v^{2} F F^{\prime \prime}\left(\partial_{\mu} h\right)\left(D_{\mu} \bar{\pi}\right)_{c} & -v^{2} F F^{\prime \prime}\left(D_{\mu} \bar{\pi}\right)^{2}
\end{array}\right], \\
& R_{j k l}^{i}\left(D_{\mu} \phi\right)^{k}\left(D_{\nu} \phi\right)^{l} \\
& =\left[\begin{array}{cc}
{\left[1-v^{2}\left(F^{\prime}\right)^{2}\right]\left[\left(D_{\mu} \bar{\pi}\right)^{a}\left(D_{\nu} \bar{\pi}\right)_{b}-\left(D_{\nu} \bar{\pi}\right)^{a}\left(D_{\mu} \bar{\pi}\right)_{b}\right]} & F^{\prime \prime} \\
F & 0
\end{array}\right] .
\end{aligned}
$$

The Lagrangian term $\mathcal{I}(\phi)$ containing the potential and Yukawa couplings is

$$
\mathcal{I}(\phi)=-V(h)+K(h) \boldsymbol{n} \cdot \boldsymbol{W}
$$


where $\boldsymbol{W}$ is a constant, in the notation of ref. [1].

$$
\nabla_{i} \nabla_{j} \mathcal{I}=\left[\begin{array}{cc}
g_{a b}\left[v^{2} F F^{\prime}\left(\boldsymbol{W} \cdot \boldsymbol{n} K^{\prime}-V^{\prime}\right)-\boldsymbol{W} \cdot \boldsymbol{n} K\right] & F\left(\frac{K}{F}\right)^{\prime} \boldsymbol{W} \cdot \boldsymbol{n}_{, a} \\
F\left(\frac{K}{F}\right)^{\prime} \boldsymbol{W} \cdot \boldsymbol{n}_{, b} & -V^{\prime \prime}+K^{\prime \prime} \boldsymbol{n} \cdot \boldsymbol{W}
\end{array}\right]
$$

where $\boldsymbol{n}_{, a}=\partial \boldsymbol{n} / \partial \bar{\pi}^{a}$.

The field strength $Y_{\mu \nu}$ is

$$
\begin{aligned}
{\left[Y_{\mu \nu}\right]_{j}^{i}=} & {\left[\begin{array}{cc}
{\left[1-v^{2}\left(F^{\prime}\right)^{2}\right]\left[\left(D_{\mu} \bar{\pi}\right)^{a}\left(D_{\nu} \bar{\pi}\right)_{b}-\left(D_{\nu} \bar{\pi}\right)^{a}\left(D_{\mu} \bar{\pi}\right)_{b}\right]} & F^{\prime \prime} \\
F & {\left[\left(\partial_{\mu} h\right)\left(D_{\nu} \bar{\pi}\right)^{a}-\left(\partial_{\nu} h\right)\left(D_{\mu} \bar{\pi}\right)^{a}\right]}
\end{array}\right] } \\
& +v^{2} F F^{\prime \prime}\left[\left(\partial_{\mu} h\right)\left(D_{\nu} \bar{\pi}\right)_{b}-\left(\partial_{\nu}\right) h\left(D_{\mu} \bar{\pi}\right)_{b}\right]
\end{aligned}
$$

with

$$
A_{\mu \nu}^{\beta}\left(t_{\beta}^{i}\right)_{; j}=\left[\begin{array}{cr}
0 & -F F^{\prime}\left(\partial_{b} n\right)^{T} \mathscr{A}_{\mu \nu} n \\
v^{2} \frac{F^{\prime}}{F} g^{a c}\left(\partial_{c} n\right)^{T} \mathscr{A}_{\mu \nu} n & g^{a c}\left(\partial_{c} n\right)^{T} \mathscr{A}_{\mu \nu}\left(\partial_{b} n\right)
\end{array}\right]
$$

and

$$
\mathscr{A}_{\mu}=\left[\begin{array}{cccc}
0 & g W_{\mu}^{3}+g^{\prime} B_{\mu} & -g W_{\mu}^{2} & g W_{\mu}^{1} \\
-g W_{\mu}^{3}-g^{\prime} B_{\mu} & 0 & g W_{\mu}^{1} & g W_{\mu}^{2} \\
g W_{\mu}^{2} & -g W_{\mu}^{1} & 0 & g W_{\mu}^{3}-g^{\prime} B_{\mu} \\
-g W_{\mu}^{1} & -g W_{\mu}^{2} & -g W_{\mu}^{3}+g^{\prime} B_{\mu} & 0
\end{array}\right]
$$

in terms of the electroweak gauge bosons. The field strength tensor $\mathscr{A}_{\mu \nu}$ is given by eq. (B.10) with the replacements $W_{\mu}^{\alpha} \rightarrow W_{\mu \nu}^{\alpha}, B_{\mu} \rightarrow B_{\mu \nu}$. The covariant derivative $D_{\mu} \boldsymbol{n}$ is given by

$$
D_{\mu} \boldsymbol{n}=\partial_{\mu} \boldsymbol{n}+\mathscr{A}_{\mu} \boldsymbol{n}
$$

treating $\boldsymbol{n}$ as a four-component column vector, and using matrix multiplication. The covariant derivative on $\bar{\pi}$ is defined implicitly through

$$
D_{\mu} \boldsymbol{n} \cdot D^{\mu} \boldsymbol{n}=g_{a b}(\bar{\pi})\left(D_{\mu} \bar{\pi}\right)^{a}\left(D_{\mu} \bar{\pi}\right)^{b}
$$

Substituting the above equations into eq. (3.43) gives eq. (59) in ref. [1].

\section{Non-reductive cosets}

In this appendix, we comment briefly on the CCWZ formalism when $\left[T_{a}, X_{B}\right]$ contains a piece proportional to the unbroken generators, so that the coset is non-reductive. Such examples are relevant for constructing $\mathcal{G} / \mathcal{H}$ theories with negative sectional curvature [1].

One can still define the CCWZ $\xi$ field as in eq. (4.9) which transforms as in eq. (4.12). The complication for the non-reductive case is in eq. (4.13). For $g \in H$,

$$
g\left(\pi^{A}(x) X_{A}\right) g^{-1}
$$


is no longer a linear combination of the broken generators, but also has a component along the unbroken generators,

$$
g \pi^{A}(x) X_{A} g^{-1}=X_{A}\left[D^{\mathbf{R}^{(\pi)}}(g)\right]^{A}{ }_{B} \pi^{B}+T_{a} M_{B}^{a} \pi^{B},
$$

where $D^{\mathbf{R}^{(\pi)}}$ is the $\mathbf{R}^{(\pi)}$ transformation matrix constructed out of $f_{a B}{ }^{C}$, as in eq. (4.6), and $M_{B}^{a} \pi^{B}$ is the component in the unbroken direction. The exponential of eq. (C.2) can be schematically written as

$$
e^{X+T}=e^{X^{\prime}} e^{T^{\prime}}
$$

where $X, X^{\prime}$ are linear combinations of broken generators, and $T, T^{\prime}$ are linear combinations of unbroken generators, and the primed and unprimed quantities are connected by the Baker-Campbell-Hausdorff formula. Thus one gets eq. (4.12) with some important changes even if $g \in H$ : (a) The relation between $\pi$ and $\pi^{\prime}$ is non-linear. Eq. (4.14) only holds for the linear term, i.e. for the transformation of the tangent vector to the Goldstone boson manifold at the origin, and (b) $h^{\prime}(\xi(x), g)$ depends on $\xi$ and hence $x$, even if $g \in H$.

The transformation of $\left(D_{\mu} \pi\right)$ and $V_{\mu}$ in eqs. (4.17) and (4.18) is also changed,

$$
\begin{gathered}
\left.\left(D_{\mu} \pi\right) \rightarrow h\left(D_{\mu} \pi\right) h^{-1}\right|_{X} \\
V_{\mu} \rightarrow h V_{\mu} h^{-1}-\partial_{\mu} h h^{-1}+\left.h\left(D_{\mu} \pi\right) h^{-1}\right|_{T}
\end{gathered}
$$

$\left(D_{\mu} \pi\right)$ transforms by adjoint action by $\mathcal{H}$ in the representation $\mathbf{R}^{(\pi)}$, as before. However, $V_{\mu}$ picks up an additional piece and no longer transforms as a gauge field under $\mathcal{H}$. One can still define Goldstone boson kinetic terms as before, eq. (4.20). However, since $V_{\mu}$ does not transform as a gauge field, it is not possible to define covariant derivatives on matter fields $\psi$ which transform as arbitrary irreducible representations of $\mathcal{H}$, as was done in CCWZ. Nevertheless, some matter fields are allowed in the EFT. For example, if $\psi$ transforms as a representation $\mathbf{R}_{G}$ of the full group $\mathcal{G}$,

$$
\psi \rightarrow D(g) \psi
$$

then

$$
\left(\partial_{\mu}+i t_{\alpha} A_{\mu}^{\alpha}\right) \psi
$$

is a covariant derivative, where the generators $t_{\alpha}$ are in the $\mathbf{R}_{G}$ representation. Following CCWZ, we can define new fields $\chi$ by

$$
\chi=D\left(\xi^{\dagger}\right) \psi
$$

which transform as

$$
\chi \rightarrow D(h) \chi
$$


where $h$ is given by eq. (4.12). The covariant derivative eq. (C.7) turns into

$$
\left(\partial_{\mu}+\xi^{-1} D_{\mu} \xi\right) \chi=\left[\partial_{\mu}+i\left(D_{\mu} \pi\right)^{A} X_{A}+i V_{\mu}^{a} T_{a}\right] \chi
$$

on $\chi$ using eq. (4.17). The sum $\left(D_{\mu} \pi+V_{\mu}\right)$ in the covariant derivative transforms as a gauge field

$$
\left(D_{\mu} \pi+V_{\mu}\right) \rightarrow h\left(D_{\mu} \pi+V_{\mu}\right) h^{-1}-\partial_{\mu} h h^{-1},
$$

and the covariant derivative eq. (C.10) is well-defined. For compact groups, where $\left(D_{\mu} \pi\right)$ transforms as

$$
\left(D_{\mu} \pi\right) \rightarrow h\left(D_{\mu} \pi\right) h^{-1}
$$

and does not mix with $V_{\mu}$, one can omit $\left(D_{\mu} \pi\right)$ in eq. (C.10) to get the CCWZ covariant derivative. In this case, for the covariant derivative on $\chi$ to make sense, it is only necessary to define the action of the unbroken generators $T_{a}$ on $\chi$, i.e. one can restrict $\chi$ to only be in an irreducible representation of $\mathcal{H}$; it does not have to form a representation of $\mathcal{G}$. Baryons in QCD are an example - they form a representation of the unbroken $\mathrm{SU}(3)_{V}$ symmetry, but not of chiral $\mathrm{SU}(3)_{L} \times \mathrm{SU}(3)_{R}$. However, for the non-reductive case, it is necessary to retain the $\left(D_{\mu} \pi\right)$ term in the covariant derivative, to cancel the extra piece in the transformation of $V_{\mu}$, the last term in eq. (C.5). In this case, we need to define the action of $T_{a}$ and $X_{A}$, which requires $\chi$ to form a representation of the full symmetry $\mathcal{G}$, not just its unbroken subgroup.

The main difficulty for sigma models with non-compact $\mathcal{H}$ is unitarity. The $\psi$ kinetic energy term for compact groups $\mathcal{H}$ is

$$
\sum_{a}\left(D_{\mu} \psi\right)_{a}^{\dagger}\left(D^{\mu} \psi\right)^{a}
$$

if $\psi$ is a complex scalar. If $\mathcal{H}$ is non-compact, then the unitary representations are infinite dimensional. For a finite dimensional non-unitary representation, the kinetic term eq. (C.13) is not an invariant, since $\psi^{\dagger}$ does not transform as the inverse of $\psi$. One can construct invariant terms. For example, if $\mathcal{H}$ is $\mathrm{SO}(3,1)$, and $\psi$ transforms as the (real) vector representation,

$$
\sum_{i=1,2,3}\left(D_{\mu} \psi_{i}\right)\left(D^{\mu} \psi_{i}\right)-\left(D_{\mu} \psi_{4}\right)\left(D^{\mu} \psi_{4}\right)
$$

is invariant, as should be familiar from the Lorentz group. Eq. (C.14) has a wrong sign kinetic term, and leads to ghosts. We do not know, in general, whether there are finite dimensional representations for a non-compact group $\mathcal{H}$ with a positive definite $\mathcal{H}$-invariant kinetic energy term. This is possible for a trivial example: if $\mathcal{H}$ is a non-compact $\mathrm{U}(1)$, i.e. of the form $h=\exp \alpha T,-\infty \leq \alpha \leq \infty$, one can pick the fermion to transform as $\exp i q \alpha$, and the kinetic energy eq. (C.13) is $\mathcal{H}$-invariant.

One can construct a suitable kinetic energy term if $\mathcal{H}$ is compact even if $\mathcal{G}$ is noncompact, since $\psi$ transforms under $\mathcal{H}$, not $\mathcal{G}$. An example of this type based on $\mathrm{SO}(4,1) \rightarrow$ 
$\mathrm{SO}$ (4) was studied in ref. [33]. In this case, the low energy EFT is unitary. However, implementing a unitary UV theory in which $\mathcal{G}$ invariance is manifest is problematic, and we do not know of any examples where this is possible. ${ }^{6}$

\section{C.1 Example of a non-reductive coset}

A simple example of a non-reductive coset is the 2-parameter group of matrices

$$
\left[\begin{array}{ll}
1 & 0 \\
x & y
\end{array}\right], \quad y>0
$$

under multiplication. The generators (absorbing a factor of $i$ ) can be chosen as

$$
T=\left[\begin{array}{ll}
0 & 0 \\
1 & 0
\end{array}\right], \quad X=\left[\begin{array}{cc}
0 & 0 \\
0 & -1
\end{array}\right],
$$

with the commutation relation

$$
[T, X]=T
$$

If the matrices act on a vector

$$
v=\left[\begin{array}{l}
0 \\
1
\end{array}\right]
$$

then $T v=0, X v \neq 0$, so that $T$ is an unbroken generator and $X$ is a broken generator. The matrices are sufficiently simple that the CCWZ formulæ can be computed explicitly. The exponential of a Lie algebra element is

$$
g=e^{a T+b X}=\left[\begin{array}{cc}
1 & 0 \\
\frac{a}{b}\left(1-e^{-b}\right) & e^{-b}
\end{array}\right],
$$

so that

$$
\xi=e^{\bar{\pi} X}=\left[\begin{array}{cc}
1 & 0 \\
0 & e^{-\bar{\pi}}
\end{array}\right]
$$

and

$$
e^{u T}=\left[\begin{array}{ll}
1 & 0 \\
u & 1
\end{array}\right]
$$

The CCWZ multiplication rule

$$
g e^{\bar{\pi} X}=e^{\bar{\pi}^{\prime} X} e^{u^{\prime} T}
$$

\footnotetext{
${ }^{6} \mathrm{~A}$ simple argument due to S. Rychkov is to look at $\mathcal{G}$-current correlators $\left\langle J_{\alpha}^{\mu} J_{\beta}^{\nu}\right\rangle$ in the UV theory. $\mathcal{G}$ invariance requires the correlator to be proportional to the Killing form $B_{\alpha \beta}$, which is not positive definite if $\mathcal{G}$ is non-compact, so that unitarity is violated. However, the low-energy EFT correlators are unitary, so it might be possible to construct theories where the $\mathcal{G}$ symmetry of $\mathcal{G} / \mathcal{H}$ arises only in the low energy limit.
} 
with $g$ in eq. (C.19) gives

$$
\begin{aligned}
\bar{\pi}^{\prime} & =\bar{\pi}+b, \\
u^{\prime} & =\frac{a}{b}\left(e^{b}-1\right) e^{\bar{\pi}} .
\end{aligned}
$$

In the special case where $g \in \mathcal{H}, b=0$ and

$$
\begin{aligned}
& \bar{\pi}^{\prime}(x)=\bar{\pi}(x), \\
& u^{\prime}(x)=a e^{\bar{\pi}(x)},
\end{aligned}
$$

so that $u^{\prime}$ depends on $x$ through $\bar{\pi}(x)$. Eq. (C.22) becomes

$$
e^{a T} e^{\bar{\pi} X}=e^{\bar{\pi} X} h, \quad h(x)=e^{a e^{\bar{\pi}}(x) T},
$$

and $h$ depends on $x$ even for an unbroken transformation.

The Maurer-Cartan form is

$$
\xi^{-1} \mathrm{~d} \xi=\mathrm{d} \pi X, \quad \omega=\left.\xi^{-1} \mathrm{~d} \xi\right|_{X}, \quad V=\left.\xi^{-1} \mathrm{~d} \xi\right|_{T},
$$

so that

$$
\omega=\mathrm{d} \bar{\pi} X, \quad V=0 .
$$

Under a global unbroken transformation $g=\exp a T$,

$$
\xi^{-1} \mathrm{~d} \xi \rightarrow \xi^{\prime-1} \mathrm{~d} \xi^{\prime}=\mathrm{d} \bar{\pi}^{\prime} X
$$

Using eq. (C.22),

$$
\omega^{\prime}=\omega, \quad V^{\prime}=0
$$

The transformation laws are

$$
\omega^{\prime}=\left.h \omega h^{-1}\right|_{X} \quad V^{\prime}=\left.h \omega h^{-1}\right|_{T}+h V h^{-1}-\mathrm{d} h h^{-1}
$$

with $h$ in eq. (C.25). These equations are satisfied because of the extra $h \omega h^{-1}$ term in the $V$ transformation.

Open Access. This article is distributed under the terms of the Creative Commons Attribution License (CC-BY 4.0), which permits any use, distribution and reproduction in any medium, provided the original author(s) and source are credited.

\section{References}

[1] R. Alonso, E.E. Jenkins and A.V. Manohar, A Geometric Formulation of Higgs Effective Field Theory: Measuring the Curvature of Scalar Field Space, Phys. Lett. B 754 (2016) 335 [arXiv: 1511.00724] [INSPIRE]. 
[2] K. Meetz, Realization of chiral symmetry in a curved isospin space, J. Math. Phys. 10 (1969) 589 [INSPIRE].

[3] J. Honerkamp and K. Meetz, Chiral-invariant perturbation theory, Phys. Rev. D 3 (1971) 1996 [INSPIRE].

[4] J. Honerkamp, Chiral multiloops, Nucl. Phys. B 36 (1972) 130 [inSPIRE].

[5] G. Ecker and J. Honerkamp, Application of invariant renormalization to the nonlinear chiral invariant pion lagrangian in the one-loop approximation, Nucl. Phys. B 35 (1971) 481 [INSPIRE].

[6] L. Álvarez-Gaumé, D.Z. Freedman and S. Mukhi, The Background Field Method and the Ultraviolet Structure of the Supersymmetric Nonlinear $\sigma$-model, Annals Phys. 134 (1981) 85 [INSPIRE].

[7] L. Álvarez-Gaumé and D.Z. Freedman, Geometrical Structure and Ultraviolet Finiteness in the Supersymmetric o-model, Commun. Math. Phys. 80 (1981) 443 [INSPIRE].

[8] D.G. Boulware and L.S. Brown, Symmetric space scalar field theory, Annals Phys. 138 (1982) 392 [INSPIRE].

[9] A. Salam and J.A. Strathdee, On Kaluza-Klein Theory, Annals Phys. 141 (1982) 316 [INSPIRE].

[10] D.H. Friedan, Nonlinear Models in Two + Epsilon Dimensions, Annals Phys. 163 (1985) 318 [INSPIRE].

[11] M.K. Gaillard, The Effective One Loop Lagrangian With Derivative Couplings, Nucl. Phys. B 268 (1986) 669 [INSPIRE].

[12] L. Alvarez-Gaume, Finite Two-Dimensional Field Theories, Comments Nucl. Part. Phys. 17 (1987) 1 [INSPIRE].

[13] J.M. Cornwall, D.N. Levin and G. Tiktopoulos, Derivation of Gauge Invariance from High-Energy Unitarity Bounds on the s Matrix, Phys. Rev. D 10 (1974) 1145 [Erratum ibid. D 11 (1975) 972] [INSPIRE].

[14] C.E. Vayonakis, Born Helicity Amplitudes and Cross-Sections in Nonabelian Gauge Theories, Lett. Nuovo Cim. 17 (1976) 383 [INSPIRE].

[15] B.W. Lee, C. Quigg and H.B. Thacker, Weak Interactions at Very High-Energies: The Role of the Higgs Boson Mass, Phys. Rev. D 16 (1977) 1519 [InSPIRE].

[16] F.-K. Guo, P. Ruiz-Femenía and J.J. Sanz-Cillero, One loop renormalization of the electroweak chiral Lagrangian with a light Higgs boson, Phys. Rev. D 92 (2015) 074005 [arXiv: 1506.04204] [INSPIRE].

[17] S.R. Coleman, J. Wess and B. Zumino, Structure of phenomenological Lagrangians. 1., Phys. Rev. 177 (1969) 2239 [INSPIRE].

[18] W. Buchmüller and D. Wyler, Effective Lagrangian Analysis of New Interactions and Flavor Conservation, Nucl. Phys. B 268 (1986) 621 [INSPIRE].

[19] B. Grzadkowski, M. Iskrzynski, M. Misiak and J. Rosiek, Dimension-Six Terms in the Standard Model Lagrangian, JHEP 10 (2010) 085 [arXiv:1008.4884] [INSPIRE].

[20] C. Grojean, E.E. Jenkins, A.V. Manohar and M. Trott, Renormalization Group Scaling of Higgs Operators and $h \rightarrow \gamma \gamma$, JHEP 04 (2013) 016 [arXiv:1301.2588] [INSPIRE]. 
[21] J. Elias-Miró, J.R. Espinosa, E. Masso and A. Pomarol, Renormalization of dimension-six operators relevant for the Higgs decays $h \rightarrow \gamma \gamma, \gamma Z$, JHEP 08 (2013) 033 [arXiv:1302.5661] [INSPIRE].

[22] J. Elias-Miro, J.R. Espinosa, E. Masso and A. Pomarol, Higgs windows to new physics through $D=6$ operators: constraints and one-loop anomalous dimensions, JHEP 11 (2013) 066 [arXiv: 1308.1879] [INSPIRE].

[23] E.E. Jenkins, A.V. Manohar and M. Trott, Renormalization Group Evolution of the Standard Model Dimension Six Operators I: Formalism and lambda Dependence, JHEP 10 (2013) 087 [arXiv:1308.2627] [INSPIRE].

[24] E.E. Jenkins, A.V. Manohar and M. Trott, Renormalization Group Evolution of the Standard Model Dimension Six Operators II: Yukawa Dependence, JHEP 01 (2014) 035 [arXiv:1310.4838] [INSPIRE].

[25] R. Alonso, E.E. Jenkins, A.V. Manohar and M. Trott, Renormalization Group Evolution of the Standard Model Dimension Six Operators III: Gauge Coupling Dependence and Phenomenology, JHEP 04 (2014) 159 [arXiv:1312.2014] [INSPIRE].

[26] R. Alonso, E.E. Jenkins and A.V. Manohar, Holomorphy without Supersymmetry in the Standard Model Effective Field Theory, Phys. Lett. B 739 (2014) 95 [arXiv:1409.0868] [INSPIRE].

[27] R. Alonso, H.-M. Chang, E.E. Jenkins, A.V. Manohar and B. Shotwell, Renormalization group evolution of dimension-six baryon number violating operators, Phys. Lett. B 734 (2014) 302 [arXiv:1405.0486] [INSPIRE].

[28] D.B. Kaplan and H. Georgi, SU(2) × U(1) Breaking by Vacuum Misalignment, Phys. Lett. B 136 (1984) 183 [INSPIRE].

[29] M.J. Dugan, H. Georgi and D.B. Kaplan, Anatomy of a Composite Higgs Model, Nucl. Phys. B 254 (1985) 299 [INSPIRE].

[30] K. Agashe, R. Contino and A. Pomarol, The minimal composite Higgs model, Nucl. Phys. B 719 (2005) 165 [hep-ph/0412089] [INSPIRE].

[31] R. Alonso, I. Brivio, B. Gavela, L. Merlo and S. Rigolin, Sigma Decomposition, JHEP 12 (2014) 034 [arXiv:1409.1589] [INSPIRE].

[32] I.M. Hierro, L. Merlo and S. Rigolin, Sigma Decomposition: The CP-Odd Lagrangian, JHEP 04 (2016) 016 [arXiv: 1510.07899] [INSPIRE].

[33] R. Alonso, E.E. Jenkins and A.V. Manohar, $\sigma$-models with Negative Curvature, Phys. Lett. B 756 (2016) 358 [arXiv: 1602.00706] [INSPIRE].

[34] A.C. Longhitano, Heavy Higgs Bosons in the Weinberg-Salam Model, Phys. Rev. D 22 (1980) 1166 [INSPIRE].

[35] A.C. Longhitano, Low-Energy Impact of a Heavy Higgs Boson Sector, Nucl. Phys. B 188 (1981) 118 [INSPIRE].

[36] T. Appelquist and C.W. Bernard, Strongly Interacting Higgs Bosons, Phys. Rev. D 22 (1980) 200 [INSPIRE].

[37] T. Appelquist and C.W. Bernard, The Nonlinear $\sigma$ Model in the Loop Expansion, Phys. Rev. D 23 (1981) 425 [INSPIRE]. 
[38] F. Feruglio, The chiral approach to the electroweak interactions, Int. J. Mod. Phys. A 8 (1993) 4937 [hep-ph/9301281] [INSPIRE].

[39] B. Grinstein and M. Trott, A Higgs-Higgs bound state due to new physics at a TeV, Phys. Rev. D 76 (2007) 073002 [arXiv:0704.1505] [INSPIRE].

[40] B.M. Gavela, E.E. Jenkins, A.V. Manohar and L. Merlo, Analysis of General Power Counting Rules in Effective Field Theory, arXiv:1601.07551 [INSPIRE].

[41] S. Weinberg, Phenomenological Lagrangians, Physica A 96 (1979) 327 [InSPIRE].

[42] J. Gasser and H. Leutwyler, Chiral Perturbation Theory to One Loop, Annals Phys. 158 (1984) 142 [INSPIRE].

[43] A. Manohar and H. Georgi, Chiral Quarks and the Nonrelativistic Quark Model, Nucl. Phys. B 234 (1984) 189 [INSPIRE].

[44] R. Alonso, M.B. Gavela, L. Merlo, S. Rigolin and J. Yepes, The Effective Chiral Lagrangian for a Light Dynamical "Higgs Particle", Phys. Lett. B 722 (2013) 330 [Erratum ibid. B 726 (2013) 926] [arXiv:1212.3305] [INSPIRE].

[45] R. Alonso, M.B. Gavela, L. Merlo, S. Rigolin and J. Yepes, Flavor with a light dynamical "Higgs particle", Phys. Rev. D 87 (2013) 055019 [arXiv:1212.3307] [InSPIRE].

[46] G. Buchalla, O. Catà and C. Krause, Complete Electroweak Chiral Lagrangian with a Light Higgs at NLO, Nucl. Phys. B 880 (2014) 552 [arXiv:1307.5017] [InSPIRE].

[47] M.B. Gavela, J. Gonzalez-Fraile, M.C. Gonzalez-Garcia, L. Merlo, S. Rigolin and J. Yepes, CP violation with a dynamical Higgs, JHEP 10 (2014) 44 [arXiv:1406.6367] [INSPIRE].

[48] I. Brivio, J. Gonzalez-Fraile, M.C. Gonzalez-Garcia and L. Merlo, The complete HEFT Lagrangian after the LHC Run I, Eur. Phys. J. C 76 (2016) 416 [arXiv:1604.06801] [INSPIRE].

[49] I. Brivio, T. Corbett, O.J.P. É boli, M.B. Gavela, J. Gonzalez-Fraile, M.C. Gonzalez-Garcia et al., Disentangling a dynamical Higgs, JHEP 03 (2014) 024 [arXiv:1311.1823] [INSPIRE].

[50] I. Brivio, O.J.P. É boli, M.B. Gavela, M.C. Gonzalez-Garcia, L. Merlo and S. Rigolin, Higgs ultraviolet softening, JHEP 12 (2014) 004 [arXiv: 1405.5412] [INSPIRE].

[51] R. Jackiw, Functional evaluation of the effective potential, Phys. Rev. D 9 (1974) 1686 [INSPIRE].

[52] M.B. Gavela, K. Kanshin, P.A.N. Machado and S. Saa, On the renormalization of the electroweak chiral Lagrangian with a Higgs, JHEP 03 (2015) 043 [arXiv:1409.1571] [INSPIRE].

[53] L.H. Chan, Effective action expansion in perturbation theory, Phys. Rev. Lett. 54 (1985) 1222 [Erratum ibid. 56 (1986) 404] [INSPIRE].

[54] L.-H. Chan, Derivative Expansion for the One Loop Effective Actions With Internal Symmetry, Phys. Rev. Lett. 57 (1986) 1199 [InSPIRE].

[55] A. Drozd, J. Ellis, J. Quevillon and T. You, The Universal One-Loop Effective Action, JHEP 03 (2016) 180 [arXiv: 1512.03003] [INSPIRE].

[56] B. Henning, X. Lu and H. Murayama, How to use the Standard Model effective field theory, JHEP 01 (2016) 023 [arXiv: 1412.1837] [InSPIRE]. 
[57] G. 't Hooft, An algorithm for the poles at dimension four in the dimensional regularization procedure, Nucl. Phys. B 62 (1973) 444 [INSPIRE].

[58] D.V. Vassilevich, Heat kernel expansion: User's manual, Phys. Rept. 388 (2003) 279 [hep-th/0306138] [INSPIRE].

[59] C.G. Callan Jr., S.R. Coleman, J. Wess and B. Zumino, Structure of phenomenological Lagrangians. 2., Phys. Rev. 177 (1969) 2247 [InSPIRE].

[60] A. Urbano, Remarks on analyticity and unitarity in the presence of a Strongly Interacting Light Higgs, JHEP 06 (2014) 060 [arXiv:1310.5733] [INSPIRE].

[61] A.V. Manohar, Effective field theories, Lect. Notes Phys. 479 (1997) 311 [hep-ph/9606222] [INSPIRE].

[62] K. Nomizu, Invariant affine connections on homogeneous spaces, Am. J. Math. 76 (1954) 33.

[63] G.B. Gelmini and M. Roncadelli, Left-Handed Neutrino Mass Scale and Spontaneously Broken Lepton Number, Phys. Lett. B 99 (1981) 411 [INSPIRE].

[64] T. Eguchi, P.B. Gilkey and A.J. Hanson, Gravitation, Gauge Theories and Differential Geometry, Phys. Rept. 66 (1980) 213 [INSPIRE]. 